\title{
Striking the Right Chord: Signaling Enigma during Root Gravitropism
}

\author{
Manjul Singh ${ }^{1,2+}$, Aditi Gupta ${ }^{1,2 \dagger}$ and Ashverya Laxmi ${ }^{1 *}$ \\ ${ }^{1}$ National Institute of Plant Genome Research, New Delhi, India, ${ }^{2}$ Interdisciplinary Centre for Plant Genomics, University of \\ Delhi South Campus, New Delhi, India
}

OPEN ACCESS

Edited by:

Patrick H. Masson,

University of Wisconsin-Madison,

United States

Reviewed by:

Jie Le,

Chinese Academy of Sciences, China

Hsu-Liang Hsieh,

National Taiwan University, Taiwan

${ }^{*}$ Correspondence:

Ashverya Laxmi

ashverya_laxmi@nipgr.ac.in

these authors have contributed equally to this work.

Specialty section:

This article was submitted to Plant Physiology,

a section of the journal

Frontiers in Plant Science

Received: 20 March 2017

Accepted: 11 July 2017

Published: 27 July 2017

Citation:

Singh M, Gupta A and Laxmi A (2017) Striking the Right Chord: Signaling Enigma during Root

Gravitropism.

Front. Plant Sci. 8:1304. doi: 10.3389/fpls.2017.01304
Plants being sessile can often be judged as passive acceptors of their environment. However, plants are actually even more active in responding to the factors from their surroundings. Plants do not have eyes, ears or vestibular system like animals, still they "know" which way is up and which way is down? This is facilitated by receptor molecules within plant which perceive changes in internal and external conditions such as light, touch, obstacles; and initiate signaling pathways that enable the plant to react. Plant responses that involve a definite and specific movement are called "tropic" responses. Perhaps the best known and studied tropisms are phototropism, i.e., response to light, and geotropism, i.e., response to gravity. A robust root system is vital for plant growth as it can provide physical anchorage to soil as well as absorb water, nutrients and essential minerals from soil efficiently. Gravitropic responses of both primary as well as lateral root thus become critical for plant growth and development. The molecular mechanisms of root gravitropism has been delved intensively, however, the mechanism behind how the potential energy of gravity stimulus converts into a biochemical signal in vascular plants is still unknown, due to which gravity sensing in plants still remains one of the most fascinating questions in molecular biology. Communications within plants occur through phytohormones and other chemical substances produced in plants which have a developmental or physiological effect on growth. Here, we review current knowledge of various intrinsic signaling mechanisms that modulate root gravitropism in order to point out the questions and emerging developments in plant directional growth responses. We are also discussing the roles of sugar signals and their interaction with phytohormone machinery, specifically in context of root directional responses.

Keywords: Arabidopsis, gravitropism, phytohormones, glucose, signaling, root

\section{EVENTS DURING GRAVIRESPONSE IN PLANTS}

The gravitropic response mechanism can be divided into several sequential components, including perception of the change in the gravity vector, transduction, and asymmetrical growth response. Unlike unilateral light, gravity does not form a gradient between the upper and lower sides of an organ. All parts of the plant experience the gravitational stimulus equally. The first step of gravitropism addresses how do plant cells detect gravity? Two hypotheses have been proposed to explain how the direction of gravity is perceived by plants: (1) the gravitational-pressure model and (2) the starch-statolith hypothesis. The latter has been strongly supported by a variety of experimental approaches in various plant species. The second component of the gravitropic response mechanism is transduction, in which the development of hormone asymmetry is 
obtained.In the third step, a curvature response is established that allows the organ to resume growth at a defined set angle from the gravity vector; the gravitational set point angle (GSA). GSA is defined as the angle with respect to the gravity vector at which an organ is maintained as a result of gravitropism (Digby and Firn, 1995). The gravity vector $\left(\mathrm{GSA}=0^{\circ}\right.$ ) helps decide the GSA values of different organs, if an organ is maintained vertically and grows downward towards gravity vector it has a GSA of $0^{\circ}$ (e.g., a primary root) while an organ growing vertically upward against the gravity vector will have a GSA of $180^{\circ}$ (e.g., a primary shoot). Any organ growing at non-vertical angles will have a GSA between these two extremes (Digby and Firn, 1995). For a plant organ to guide its growth along a defined GSA, it must perceive any change in its orientation within the gravity field.

\section{Gravity Perception}

Plant roots are simple structure divided into various sections like root cap, meristem, elongation zone and maturation zone (Figure 1). The root cap protects root tip from obstacles in the soil and at the same time act as a guiding sensor for directional growth. There are usually four layers of columella cells at the root tip named S1-S4 (towards root tip), S1 and S2 are important for root gravitropism (Blancaflor et al., 1998). Amyloplasts that function as gravity sensors are called statoliths. These statoliths are of sufficiently high density relative to the cytosol thereby readily sediment to the bottom of the cell. The specialized gravity-sensing cells in which statoliths occur are called statocytes. The statocytes are cells with small vacuoles and a cortical ER, also their nucleus is positioned toward the shoot-ward side (Leitz et al., 2009) (Figure 2). Cortical microtubules and actin microfilaments contribute to development and maintenance of this polarity, whereas the lack of endoplasmic microtubules and prominent bundles of actin microfilaments probably facilitates sedimentation of statoliths (Volkmann and Baluska, 1999).

According to the starch-statolith hypothesis (Haberlandt, 1900) gravity reorientation relocates the amyloplasts to the new basal side of the cells (Figure 2). Sedimentation of amyloplasts towards gravity vector acts as a signal to plants which is then translated to biochemical signal that evoke the gravitropic response. In literature, the starch-statolith hypothesis of gravity sensing is supported extensively (Morita, 2010). Investigations on starchless mutant phosphoglucomutase (pgm) of Arabidopsis lacking a starch synthesis enzyme showed that they are still gravitropic but their gravity response was strongly reduced (Kiss et al., 1996). Also, ablation of columella cells through lasers or genetic means greatly reduces graviresponse (Blancaflor et al., 1998; Tsugeki and Fedoroff, 1999). Support for starch-statolith theory also comes from the use of high gradient magnetic fields to displace statoliths. Magnetophoretic displacement of statoliths causes the roots to curve toward the direction of magnetic field while hypocotyls move away from the magnetic field (Kuznetsov and Hasenstein, 1996, 1997). Changes in density of amyloplasts also affect graviresponse, the sex1 (starch excess) mutant in Arabidopsis, which is impaired in degradation of starch, accumulates excess starch in various tissues (Vitha et al., 2007). The gravitropic sensitivity of sex1 mutant roots was comparable to that of wild type (WT) roots. However, in hypocotyls, the sex 1 mutant was much more sensitive towards gravity stimulus as compared to the WT (Vitha et al., 2007). Recently, a study on gravity induced distribution of DII-Venus in pgm1 mutant provided a link between statolith sedimentation and auxin redistribution (Band et al., 2012). Also, a sudden change in amyloplast sedimentation was shown to cause differential distribution of auxin on either side of the root (Baldwin et al., 2013). These results confirm a crucial role of statoliths as a sensor of gravistimuli in plants. The elongation zone is also a critical region for studying directional movements because it is highly plastic in nature and during gravitropism, when seedlings are oriented towards their side, the visible bending is observed in the elongation zones (Miller et al., 2007). The anatomy and morphology of the graviresponding organs indicates a spatial separation between signal perception, signal formation, signal transduction and differential growth (Tasaka et al., 1999). Simple bending curvature of plants in response to change in direction of gravity vector thus becomes a complicated process.

Apart from the support provided for starch-statolith theory, there are enough clues for the existence of alternate mechanisms of gravity sensing in plants. These secondary mechanisms are supposed to be independent of starch and might also govern gravitropic bending. For example, the pgm mutants, having greatly attenuated gravitropic response, could eventually reorient their roots downward and stems upwards. (Mullen et al., 2000) have used a ROTATO device to study gravitropic response; this device holds selected region of roots at specific angles from the gravity vector on a motorized stage with an automated camera attached to it. ROTATO allows the root cap of a graviresponding root to maintain vertical non-stimulated orientation while any selected region within the elongation zone can be maintained at a gravistimulated angle. The pgm mutants responded at constant rate regardless of the increase in angles, whereas the response of WT roots increased when constrained at greater angles (Wolverton et al., 2011). The pgm mutants also lacked the auxin gradient formation as visualized by DR5 reporter expression (Wolverton et al., 2011). This result suggests that, statolith sedimentation is not the only mode of gravity sensing and there is some unknown mechanism that triggers the residual root gravitropic response in the pgm mutant independent of the angle of tip orientation. ROTATO experiments have also shown that $20 \%$ of the gravity response comes from the region within the distal elongation zone and not the root tip (Wolverton et al., 2002). ROTATO can be used as an advantageous instrument to study root gravitropism as it facilitates the exposure of any section of roots at any angle not only to microgravity conditions but also to hypo-gravity conditions with some modifications (Ishikawa et al., 2007). Removal of root cap reduces the root gravitropic response but doesn't abolish it. However, roots whose root cap is removed respond differently to gravistimulation in the presence of actin-polymerization-inhibiting drugs and these decapped roots showed faster gravi-bending as compared to WT (Mancuso et al., 2006). These results suggest that roots can sense gravity outside the zone of root cap which also depends on actin. As roots, do not contain sedimenting amyloplasts 
A
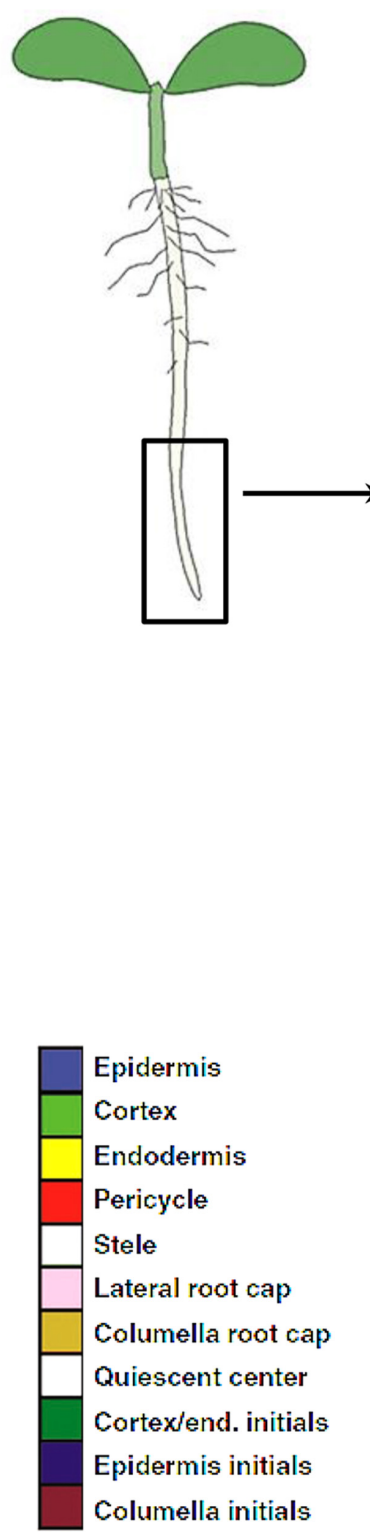

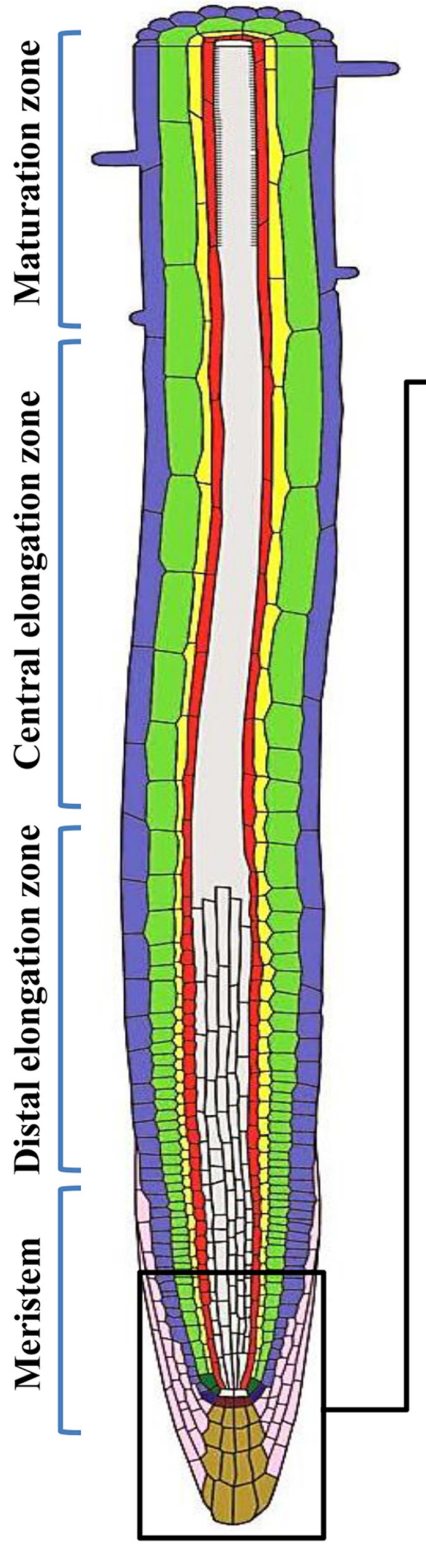

B

$\sum_{i}^{c}$
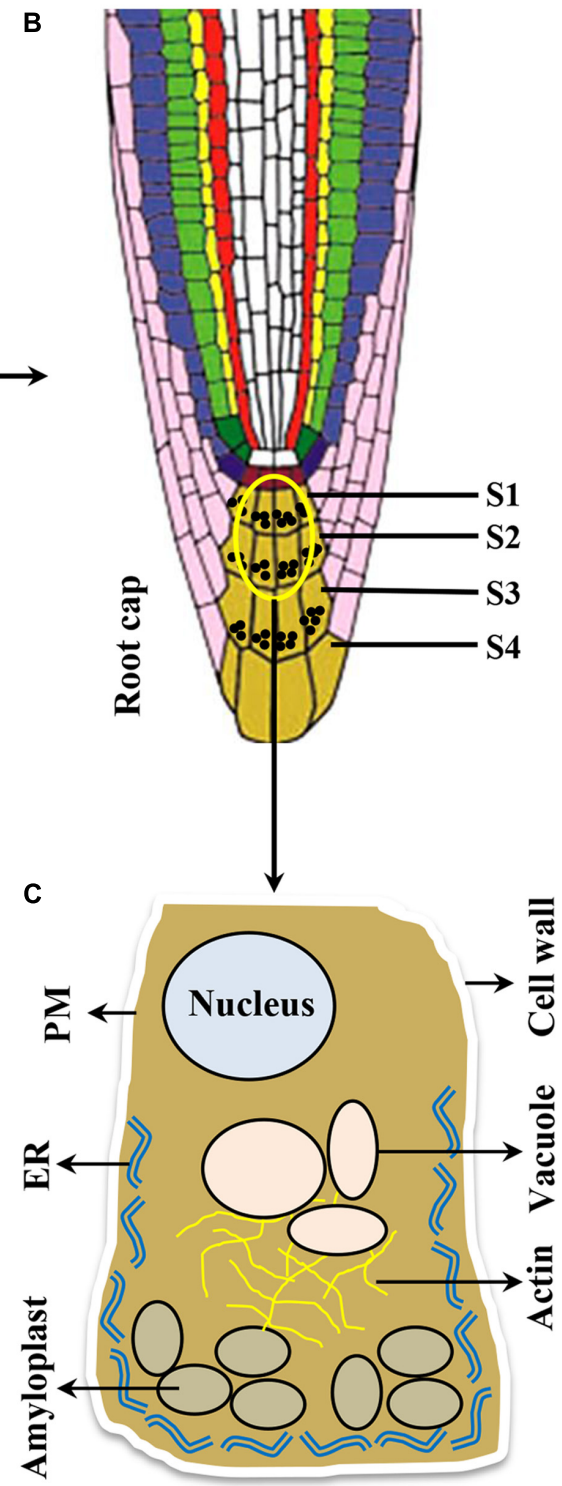

Columella cell

FIGURE 1 | The root cell types responding to gravity stimulus in Arabidopsis. (A) Longitudinal view of an Arabidopsis primary root showing meristem, Distal and central elongation zones, maturation zone. (B) Gravity sensing in roots occurs in the central columella cells of the root cap (inset). The root cap consists of four layers of cells important for root gravitropic response namely S1, S2, S3, S4, respectively. However, the central columella cells from S1 and S2 (the encircled cells) play major role for gravity sensing. (C) Columella cell (cartoon) contains starch-filled organelles called amyloplasts, nucleus, vacuole, actin filaments, endoplasmic reticulum (ER), plasma membrane (PM), and cell wall.

beyond the columella cells, therefore, the starch-statolith model might not be the only mode of gravisensing in plants (Baldwin et al., 2013). Studies on green algae Chara has provided the alternative hypothesis of gravisensing, i.e., the protoplast pressure hypothesis. Since, Chara do not contain statoliths and uses gravity as a signal to regulate their cytoplasmic streaming (Wayne et al., 1990; Staves, 1997). More studies are awaited to validate these findings and support different gravisensing hypothesis. Since root cap is major site of gravity perception, there should be some mean of communication between the gravity-sensing cells of root cap and the cells present back in the root elongation zone that ultimately respond to the gravity signal in roots.

\section{Gravity Induced Signals in Plants}

Gravity is the only constant factor, both in direction and magnitude, to which plants definitely need to- and have toadapt. Post gravity perception, a series of events takes place to transduce the signal within the plant system. With the use of electron tomography, it was found that, in response to gravity stimulus, sedimenting amyloplast can bend, and distort the 


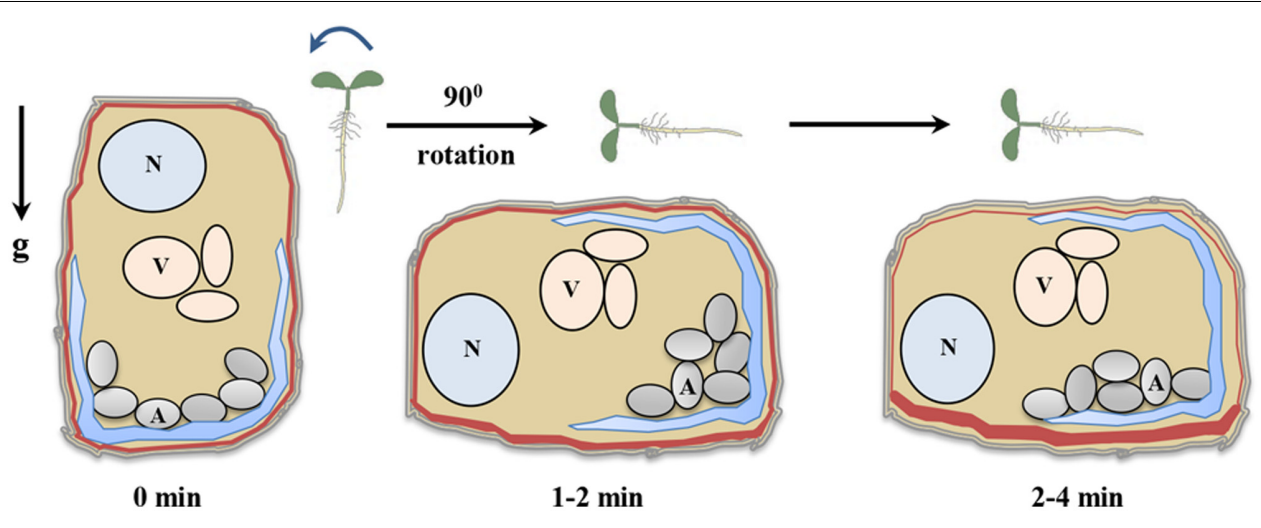

FIGURE 2 | Gravity sensing in columella cells. Roots when grown vertical have been designated time 0 min. Reorientation of seedling by rotating to $90^{\circ}$ generates a gravistimulus and initiate gravisensing process. The amyloplasts start to settle towards the new bottom of columella cells immediately post reorientation and within 2-4 min of reorientation, amyloplast settles down completely (Leitz et al., 2009). The amyloplast sedimentation creates pressure on endoplasmic reticulum membrane as a result activating mechanosensitive sites (Monshausen et al., 2011). After 2 min of reorientation, PIN3 proteins start to relocalize thereby regulating auxin flow (Friml et al., 2002). N (nucleus), V (vacuole), A (amyloplast), g (gravity), and red lines indicate PIN3 distribution.

endoplasmic reticulum (ER) upon contact (Leitz et al., 2009). The distortion of ER possibly opens stretch-activated mechanosensitive ion channels. The other hypothesis suggests a possibility of protein-protein interactions between the molecules attached to the amyloplasts and the ER due to close contact between both of them (Leitz et al., 2009). Signal transduction in gravitropism employs variety of signaling components and second messengers such as $\mathrm{Ca}^{2+}$ and $\mathrm{pH}$ (Fasano et al., 2001), actin (Blancaflor, 2013), inositol 1,4,5-triphosphate (InsP3) (Perera et al., 2006) etc. However, all these scattered findings don't give clear picture about a comprehensive pathway for gravitropism.

According to the mechano-sensitive ion channel hypothesis of signal transduction, the falling amyloplast create pressure on ER or plasma membrane either directly or via actin filaments. The pressurized ER membrane opens mechano-sensitive ion channels leading to changes in concentration of ions, such as $\mathrm{Ca}^{2+}$ which in turn leads to repolarization of statocytes, relocalization of PINs and subsequent changes in auxin transport (Baldwin et al., 2013). Gravity induced changes in $\mathrm{Ca}^{2+}$ levels are too minute to be detected. Attempts to observe changes in $\mathrm{Ca}^{2+}$ levels in the statocytes with the use of indo- 1 as the calcium sensitive detection system have not been successful in past (Legué et al., 1997). It is found that calmodulins are highly expressed in columella cells of roots (Stinemetz et al., 1987); this can be correlated with the fact that though undetected, small gravity-induced changes in intracellular $\mathrm{Ca}^{2+}$ levels could affect cell signaling. Recent advancement in sensors have helped in $\mathrm{Ca}^{2+}$ imaging technology and the detection of cytoplasmic $\mathrm{Ca}^{2+}$ changes in response to gravistimulation in both roots and shoots (Toyota and Gilroy, 2013). With the use of genetically encoded Yellow Cameleon (YC) $3.6 \mathrm{Ca}^{2+}$ biosensor, it was revealed that after gravistimulation, a wave of $\mathrm{Ca}^{2+}$ arises on the lower side of the root toward the elongation zone similar to auxin (Monshausen et al., 2011). Auxin alone is found to induce $\mathrm{Ca}^{2+}$ levels and signals (Toyota and Gilroy, 2013). Further it is known that the increase in $\mathrm{Ca}^{2+}$ levels is able to regulate auxin transport through regulation of PINOID (PID) kinase (Robert and Offringa, 2008). In addition, asymmetrical application of $\mathrm{Ca}^{2+}$ through agar block, caused root bending toward the $\mathrm{Ca}^{2+}$ source (Lee et al., 1983a). The relevance of $\mathrm{Ca}^{2+}$ signals upon gravistimulation is further substantiated with the fact that gravitropic bending is severely impaired upon chelation of apoplastic $\mathrm{Ca}^{2+}$ or by inhibition of calmodulin or $\mathrm{Ca}^{2+}$ channels (Lee et al., 1983b; Vanneste and Friml, 2013). Use of auxin transport inhibitors such as triiodobenzoicacid (TIBA) or $1-N$-naphthylphthalamic acid (NPA) has been found to block the gravity-induced $\mathrm{Ca}^{2+}$ redistribution in maize and pea roots (Lee et al., 1984). Additionally, $\mathrm{Ca}^{2+}$ dependent cross-linking of the acidic groups in pectin, $\mathrm{Ca}^{2+}$ dependent regulation of enzymes and $\mathrm{Ca}^{2+}$ modulated proteins calmodulins are also found in the extracellular matrix (Toyota and Gilroy, 2013). These findings suggest a critical role of extracellular $\mathrm{Ca}^{2+}$ in modulating gravitropic growth. Further studies are required to know others players regulating $\mathrm{Ca}^{2+}$ pumps and channels that drive these apoplastic fluxes and how amyloplast mediated perception system regulates these components.

Post gravistimulation, changes in $\mathrm{pH}$ also take place in columella cells. $\mathrm{Ca}^{2+}$ levels cause alterations in cell wall $\mathrm{pH}$ which can regulate elongation via acid growth (Monshausen et al., 2011). Changes in $\mathrm{pH}$ affect many cellular activities such as enzyme function, hormone distribution etc. Use of chemical inhibitors such as; benzoic acid, bafilomycin A1 (a vacuolar $\mathrm{H}^{+}$ATPase inhibitor) or use of caged protons to block $\mathrm{pH}$ changes caused reduction in root gravitropism but not abolished it (Scott and Allen, 1999; Fasano et al., 2001). This gives us the idea that gravistimulated changes in $\mathrm{pH}$ might also regulate the efficiency of signals generated within the gravity perceiving columella cells. In gravistimulated cells, change in $\mathrm{pH}$ can be linked with relocalization of the PIN proteins since the mutants defective in gravity induced columella cell alkalization fail to show PIN relocalization (Boonsirichai et al., 2003; Harrison and Masson, 2008). 
Mechano-sensitive channels could also open due to straining of actin filaments caused by amyloplast sedimentation as the amyloplasts are usually surrounded by a network of actin filament in the columella cells (Collings et al., 2001; Yoder et al., 2001). Surprisingly, actin destabilizing drugs like latrunculin B (Lat-b) and cytochalasin D promotes gravitropism in roots rather than inhibiting it, this way actin acts a negative regulator of gravity sensing (Blancaflor et al., 2003; Hou et al., 2004). Disruption of actin could possibly lead to more rapid sedimentation of amyloplast causing a higher compressive force on the ER, thereby adding to the overall gravity sensing and signaling potential (Yoder et al., 2001; Blancaflor, 2013; Zheng et al., 2015). With the use of microrheology analyses, amyloplast sedimentation has been studied extensively and it was found that there is abundance of actin-cytoskeletal network in the intracellular regions of central columella cells which determines the dynamics of falling amyloplasts (Zheng et al., 2015). Analysis of distorted1 (dis1) mutant whose functional protein encodes for the ARP3 subunit of the Arabidopsis Actin-Related Protein 2/3 (ARP2/3) complex revealed that dis1 mutant has disorganized thick actin bundles and showed delayed root bending upon gravity reorientation (Zou et al., 2016). The dis1pgm1 double mutant showed more delayed gravitropic curvature which was not enhanced upon Lat$B$ treatment suggesting that DIS1 is involved in gravity sensing as well as signal transduction (Zou et al., 2016). Further, it was found that ARP3/DIS1 was involved in asymmetric auxin redistribution via modulation of PIN cycling (Zou et al., 2016).

Possible physical interactions between the sedimenting amyloplast and the ER bound proteins can also be involved in generating gravity signals within cells. The ligand-receptor hypothesis came from the study of single-celled rhizoids of the green algae Chara. In Chara, the statoliths are barium-sulfatefilled vesicles. During parabolic flights the weightless statoliths were able to trigger gravity signal as long as there was a contact with sensitive sites in plasma membrane (Limbach et al., 2005). It was then proposed that, in Chara rhizoids, pressure exerted by statoliths weight was not responsible for gravity sensing, rather it was dependent upon direct contacts between components present on statoliths and membrane-bound receptors (Braun and Limbach, 2006). Similar type of phenomenon may also exist in plants and can be correlated with the fact that reduced gravitropic sensitivities in starchless mutant can be restored by sedimenting their plastids through hypergravity (Fitzelle and Kiss, 2001). This hypothesis was further substantiated with the screens for various mutants showing altered gravitropic responses. The Arabidopsis mutant altered response to gravity1 (arg1) showed reduced root and hypocotyl gravitropism (Sedbrook et al., 1999). The ARG1 gene encodes a DNA-J like protein with conserved J domain and a coiled-coil region (Sedbrook et al., 1999). ARG1 has two paralogs; ARG-like1 (ARL1) and ARL2, of which arl2 mutants showed gravitropic defects very similar to $\operatorname{arg1}$. Both ARG1 and ARL2 operate in the same pathway. ARG1 localizes to the endomembrane system, whereas ARL2 is mainly found associated with the plasma membrane and selectively expressed in gravisensing cells (Boonsirichai et al., 2003; Harrison and Masson, 2008). To find out genetic enhancers of arg1, researchers used forward genetics approach and isolated two mutants; modifier of arg1 (mar1) and mar2. MAR1 and MAR2 belong to the Translocon of the Outer envelope of Chloroplasts (TOC) complex and encode for two different components TOC75 and TOC132, respectively, in Arabidopsis (Stanga et al., 2009). The TOC complexes consist of a pore (Toc75/MAR1); Toc159 family receptors (TOC159, TOC132/MAR2, TOC120, or TOC90); and a TOC34 family receptor (TOC33 or TOC34/PPI3). The TOC complex helps in delivery of nuclear encoded proteins to chloroplasts (Strohm et al., 2014). ARG1 and the TOC complex have different functions and are differentially localized. The mar2 single mutant has no obvious gravitropic phenotype while arg1mar2 double mutants are agravitropic (Stanga et al., 2009). The columella cell amyloplasts are also normal in arg1mar2 double mutants; they contain normal amounts of starch and have normal sedimentation kinetics (Stanga et al., 2009). Why the corresponding mutations show a strong genetic interaction within the gravitropism signaling raises points for the ligand-receptor hypothesis. TOC132 act as a receptor on plastid surface for preprotein recognition during translocation (Perry and Keegstra, 1994). This finding fits well with the hypothesis that TOC132 would play a role in mediating plastid interactions with proteins located on the cortical ER or plasma membrane during gravistimulation (Baldwin et al., 2013).

Another signaling molecule involved in graviresponse is Inositol 1,4,5-trisphosphate (InsP3). Evidence for the role of InsP3 in gravitropism came from measurement of InsP3 levels during early gravitropic responses. Upon gravistimulation, InsP3 fluxes were found to first fluctuate and then increase at the bottom half of oat pulvinus (Perera et al., 2001). Similar results were also observed in case of Arabidopsis stem (Perera et al., 2006). The Arabidopsis root, stem and hypocotyl showed gravitropic defects upon constitutively overexpressing human TYPE I INOSITOL POLYPHOSPHATE 5-PHOSPHATASE (InsP 5-ptase) which specifically hydrolyses InsP3 (Perera et al., 2006). Activation of PHOSPHOLIPASE C (PLC) leads to an increase in inositol 1,4,5-triphosphate ( $\mathrm{InsP}_{3}$ ) levels. Microarray studies conducted on plants, manipulated in their InsP3 metabolism by inhibiting PLC activity or overexpressing InsP 5-ptase, identified $\mathrm{InsP}_{3}$-dependent and independent co-regulated genes in response to gravity (Salinas-Mondragon et al., 2010). In Arabidopsis, INOSITOL POLYPHOSPHATE 5-PHOSPHATASE 13 gene (5PTase13) encodes for an enzyme involved in breaking down of InsP3. The knockout 5 pt13 mutant has higher levels of InsP3 and enhanced root gravitropic responses (Wang et al., 2009). The 5pt13 mutant shows reduced response to auxin transport inhibitor NPA, suggesting an increased polar auxin transport (Wang et al., 2009). In another study, it was found that PHOSPHATIDYLINOSITOL MONOPHOSPHATE 5-KINASE (PIP5K), a key enzyme in the phosphatidylinositol pathway, is involved in gravitropism. In phosphatidylinositol pathway, PIP5K catalyzes synthesis of PI-4,5-bisphosphate, a precursor of secondary messengers InsP3, and diacylglycerol (DAG) (Mei et al., 2012). The knockout pip5k2 mutant shows delayed root gravity response (Mei et al., 2012). The pip5k2 mutants are more sensitive to NPA as compared to WT, suggesting that it has impaired polar auxin transport (Mei et al., 2012). Recently, a root-specific protein named InteractoR Of SYnaptotagmin1 
(ROSY1) has been identified as one of the earliest transcribed signals during root gravitropic response in Arabidopsis (Dalal et al., 2016). ROSY1 accumulates on the upper side of the gravistimulated root and rosy1-1 mutant roots displayed faster gravitropic bending. ROSY1, is a stigmasterol binding protein which interacts with a membrane transport and recycling protein, synaptotagmin-1 and thus might affect vesicle recycling during the asymmetrical gravitropic growth (Dalal et al., 2016).

\section{Other Directional Responses in Plants}

Root growth is governed by the coordinated events of cell division and elongation of the newly formed cells. Plants roots are extremely sensitive to environmental stimulation such as; gravity, mechanical obstacles, light, moisture and nutrient gradients modulating the directional growth of roots to obtain an optimal growth trajectory. The initial work of Darwin represented various plant movements which were defined as a result of circumnutation (Darwin and Darwin, 1880). Plant movements are categorized in various categories such as "tropic movement" induced by a constant directional factor like gravity or light and "nastic movement" induced by an external factor but independent of direction of stimulus. Circumnutation is the circular or elliptical movement attained by plant organs while growing through a particular direction. Nutations are organ movements created due to unequal growth rate on two sides of an organ and are governed mainly by internal factors (Migliaccio et al., 2013). Out of these movements, tropic and nastic movement mostly occur together in plants. These movements produce various growth patterns in roots based on the substrate and environment. Among these growth patterns produced in roots are; root-waving, -coiling and -skewing etc. When Arabidopsis seedlings are grown on slanted high agar density (1.5-2\%) containing media they show waving and skewing pattern of root growth. This kind of growth behavior is a result of touch, gravity and circumnutation (Migliaccio and Piconese, 2001). Waving is not apparently seen when the agar plates are set vertically. Once the plates are slanted, root experience gravity and tries to move downward but unable to penetrate the hard medium which results in a forced response towards a touch stimulus. This cumulative stimulus of touch and gravity when accumulated with circumnutation gives rise to rootwaving. However, studies evaluating the role of environment and nutritional conditions further complicated our understanding of this process (Buer et al., 2000). Another peculiar aspect of Arabidopsis root growth, normally visible in commonly used ecotypes like Landsberg (Ler), Wassilewskija (Ws) and Columbia (Col), in which roots tend to move in a particular direction when grown vertically is termed as skewing (Simmons et al., 1995). The intensity of the slanting differs among the accessions and in some way might be connected with the waving movements. The root skewing was further studied and it was observed that the observed rotation of root tip was an outcome of epidermal cell file rotation (CFR) (Yuen et al., 2005). The slanting movement is a result of touch stimuli and twisting of Arabidopsis roots. It should be taken into account that waving and skewing are visible on the surface and do not appear when a root grows within the medium because inside the medium root experiences a uniform touch/pressure around the growing root. Skewing is quantified as rightward or leftward based on the slant angle on the medium whereas waving is usually quantified by their frequency and amplitude of waves. Coiling is another kind of movement shown by Arabidopsis roots when grown on horizontal plates. The coils formed are always right handed in WT with roots showing left handed torsion (Mirza, 1987). The morphology of coils, varies depending on the nutritional quality and conditions of illumination (Mirza, 1987). Taken together, analysis of waving, skewing and coiling in addition to tropic responses can be a good system to study the dynamics of root growth and movement, however the actual root growth movement in the soil is different and any conclusions drawn based on root-gel/medium interactions need further confirmation in the soil system.

\section{PHYTOHORMONES AND GRAVIRESPONSES IN PLANTS}

Phytohormones have profound effects on development at vanishingly low concentrations. The emerging concept of cooperative hormone action opens new possibilities for a better understanding of the complex interactions between all phytohormones and their possible synergistic effects on regulation of gravitropism. Even though numerous reports on gravitropism are published, the actual gravity receptor has not been identified yet. Auxin, ethylene, cytokinin and BRs have been the most explored hormones in relation to gravitropism but not much evidence has been accumulated regarding the participation of other phytohormones such as; Gibberellins (GAs), abscisic acid (ABA), jasmonates (JA), and salicylic acid (SA) in gravitropism.

\section{Auxin as an Essential Player in Root Gravitropism}

Auxin was the earliest hormone to be identified with an implicated function during gravitropism, and for many years it dominated as the primary hormone regulating graviresponses. Auxin transport and response to auxin is pre-requisite for the development of tropic curvatures. Auxin, which is mainly synthesized in young shoot tissues, uses a cell-to-cell transport system that functions in the tip to base direction in shoots. When auxin reaches the root, it is transported through the central cylinder into root tip, where it adds to a pool of locally synthesized auxin, forming an auxin-maximum center that overlaps with the quiescent center and top layers of the root-cap columella. There, auxin is redistributed laterally to peripheral tissues, then transported basipetally through lateral-cap and epidermal cell files toward the elongation zone, where it inhibits cell elongation (Figure 3). Upon gravity reorientation, auxin accumulates in the lower region of the roots, as a result the cells of upper region elongate more and gravistimulated roots display a downward curvature. Modulation of auxin transport is therefore crucial for auxin redistribution and formation of an auxin gradient across a gravistimulated organ (Friml, 2003; Vanneste and Friml, 2009). The role of auxin transport in gravitropism was further understood by extensive studies examining the effect of various auxin transport inhibitors on the gravitropic response and also 


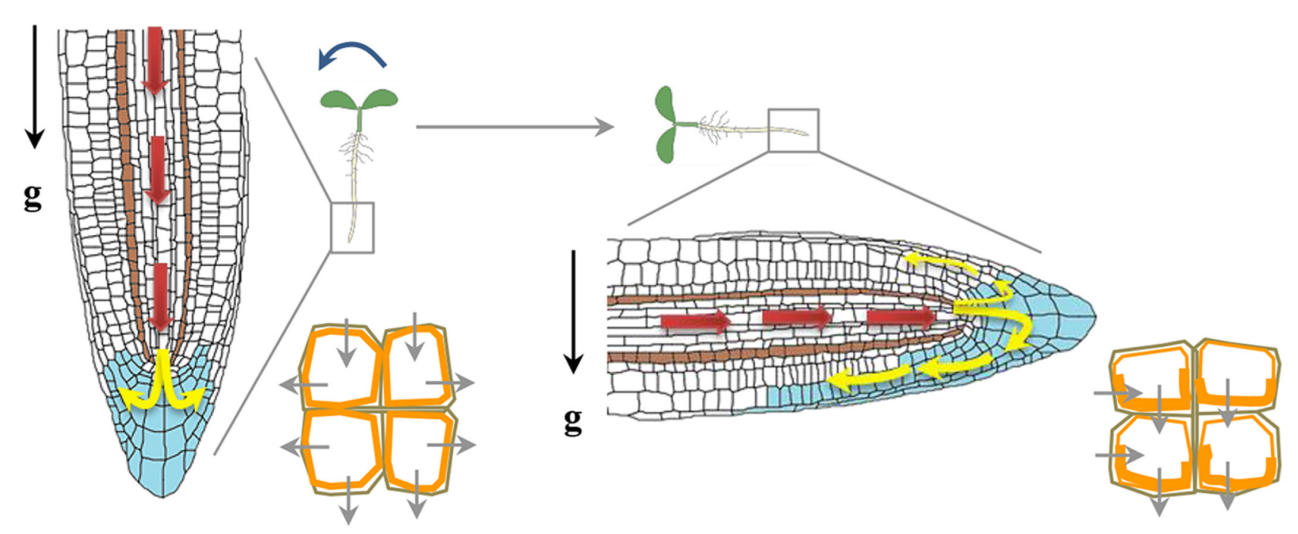

FIGURE 3 | Auxin redistribution upon a gravity stimulus. Auxin distribution (blue) and direction of flow (depicted by arrows). Auxin from the shoot to the root tip (red arrows) is mediated by AUX1 and PIN2. Auxin flow is further distributed through the vascular tissue to the columella cells (yellow arrows). PIN3 is localized in the columella cells and gravity stimulus induces more PIN3 localization (orange) to the lower side of columella cells redirecting auxin flow to the lower side of the root (Friml et al., 2002). Asymmetric localization of PIN2 upon gravity reorientation too helps auxin flow at the lower side of gravistimulated root mainly directed towards the elongation zone, where it inhibits elongation at the lower side of the root and causes root bending.

by showing that mutations in genes encoding key components of auxin transport machinery altered root gravitropism.

There are three types of transmembrane proteins that mainly transport auxin across the plasma membrane; (i) the transmembrane proteins AUXIN RESISTANT 1/LIKE AUX1 (AUX1/LAX), which operates as influx carriers to enable the transport of protonated auxin (Swarup and Péret, 2012); (ii) PIN-FORMED (PIN) proteins acting as auxin efflux carriers and (iii) members of the MULTIDRUG-RESISTANT (MDR)/PGLYCOPROTEIN (PGP) family of ATP-binding cassette (ABC) transporters which are auxin efflux transporters. Mutation in AUX1 causes disruption of the basipetal auxin transport from the shoot tip to the root tip causing a reduction in the gravitropic root response of the mutant. Using fluorescent $\mathrm{pH}$ sensors, a marked increase in the surface $\mathrm{pH}$ on the lower side of a gravistimulated root was observed in WT but not in aux1 mutant (Monshausen et al., 2011). This finding suggests that AUX1 is important in root gravitropism as the increase in root apoplastic $\mathrm{pH}$ results in more auxin in its ionic $\mathrm{IAA}^{-}$form which is not permeable and requires AUX1 as a carrier (Swarup and Péret, 2012). Several lines of evidence strongly support a role of PIN auxin efflux carriers in gravitropism. In roots, PIN3, 4 and 7 are localized in columella cells (Sato et al., 2015). The PIN3 protein is expressed in upper S1 and S2 layers of the columella cells, whereas PIN7 protein is found in the S2 and S3 layers where they regulate auxin gradient immediately upon gravistimulation across the root cap (Kleine-Vehn et al., 2010). In the absence of PIN3, PIN7 expands its expression into the S1 layer and compensate for the loss of PIN3 (Kleine-Vehn et al., 2010). In vertically growing roots, these PINs show non-polar localization whereas upon gravistimulation, they are endocytosed and localized to the lower side of plasma membrane; thereby generating lateral auxin gradient across the root cap (Strohm et al., 2012). The PIN3 protein is recycled in an actin dependent manner (Friml et al., 2002). The involvement of PIN3 and PIN7 in gravitropism is substantiated by the fact that pin 3 and pin 7 mutants show gravitropic defects, and the pin3 pin7 double mutant shows even stronger defects than their respective single mutants (Friml et al., 2002; Kleine-Vehn et al., 2010). The pin2 mutant is agravitropic and the auxin distribution upon gravity stimulation is perturbed in pin2 background (Luschnig et al., 1998). The polarity of PIN proteins is regulated by intracellular trafficking. Small GTPases (small G proteins) and GDP/GTP exchange factor (GEF) proteins regulate the rootward localization of PINS. GNOM which is a GEF, modulate the activity of a small G-protein named ADP ribosylation factor (ARF) and affects the localization of PIN3 proteins (Strohm et al., 2012). BrefeldinA (BFA) inhibits trafficking of PIN3 by inhibiting GNOM, as a result PIN3 endocytosis continues but they get trapped in intracellular compartments (Kleine-Vehn et al., 2010). Engineering GNOM proteins in such a way that they become BFA insensitive (Geldner et al., 2003), lead to proper localization of PIN1 and PIN3, proper auxin gradients after gravistimulation and normal gravitropism even in presence of BFA (Geldner et al., 2003; Kleine-Vehn et al., 2010). Another gravitropism responsive gene identified by forward genetics approach is SPIKE1 (SPK1). SPK1 is a GEF for Rho-like GTPase from Plants 6 (ROP6). The spk1 mutants show slow gravitropic response and decreased PIN2 levels at the plasma membrane, whereas overexpression of ROP6 causes an increased gravitropic response (Lin et al., 2012). SPIKE1 binds to the inactive form of ROP6 and inhibit PIN2 endocytosis in roots (Lin et al., 2012). The polar localization of PIN protein is also dependent upon their phosphorylation status as use of kinase inhibitors has been shown to disrupt localization of PIN2 (Sukumar et al., 2009). Phosphorylation by PID kinase and dephosphorylation by a TYPE 2A PROTEIN PHOSPHATASE (PP2A), ROOTS CURL IN NAPHTHYLPHTHALAMIC ACID1 (RCN1) act antagonistically to each other and regulate auxin transport and gravitropism (Sukumar et al., 2009). The continuous recycling of PIN proteins helps in the dynamic control of polar localization and abundance at the plasma membrane which ultimately 
determines the rate and directionality of auxin flow (Baster et al., 2013). Recently, the R2R3-MYB type transcription factors FOUR LIPS (FLP) and its paralogue MYB88 has been reported to regulate transcript abundance of PIN3 and PIN7 in the gravity sensing cells of primary as well as lateral roots of Arabidopsis (Wang et al., 2015). The fact that most of the mutations in auxin transporters caused impaired root gravitropism strengthens the credit for auxin transport to play a more important role in gravitropism. However, the auxin gradient across the gravistimulated organ is transient and auxin asymmetry does not persist during the whole length of the gravitropic response. Such a temporal auxin asymmetry seems to be responsible for activation of auxin-responsive genes and subsequent downstream events leading to the bending response.

Analysis of gravitropic response of mutants defective in auxin-signaling provided additional support for the involvement of auxin response pathway during root gravitropism. Upon gravistimulation, many auxin-responsive genes are differentially regulated. Auxin is sensed by members of TRANSPORT INHIBITOR RESPONSE 1/AUXIN-RELATED F-BOX (TIR1/AFB) family of auxin receptors (Parry et al., 2009). AUXIN RESPONSE FACTORS (ARFs) family of transcription factors bind to auxin-responsive cis-acting elements (AuxREs) present in auxin induced genes (Remington et al., 2004). The AUXIN/INDOLE-3-ACETIC ACID INDUCIBLE (AUX/IAA) proteins are auxin induced nuclear localized short-lived repressor proteins. Under low auxin concentration, ARFs form dimers with AUX/IAAs, thereby shutting down transcription of auxin regulated genes. The repression of transcription by AUX/IAAs is also facilitated by TPLs (Szemenyei et al., 2008). Upon auxin accumulation, AUX/IAA is rapidly degraded executed via active E3 ligase SKP-Cullin-F-box TRANSPORT INHIBITOR RESPONSE 1 $\left(\mathrm{SCF}^{\mathrm{TIR} 1}\right)$ complex and this process allows ARF-ARF dimerization and transcription activation of target genes (Leyser, 2002). Mutations in the AUX/IAA genes; IAA3, IAA7, IAA17 and IAA14 conferred abnormal gravitropic response in the hypocotyls and roots of their respective mutants short hypocotyl 2 (shy2), auxin resistant 2 (axr2-1), axr3 and solitary root (slr-1) respectively (Leyser et al., 1996; Tian and Reed, 1999; Fukaki et al., 2002; Masson et al., 2002). The auxin-insensitive tir1, axr6, and axr 1 mutants also exhibited a reduced gravitropic response in roots (Masson et al., 2002). The arf7 and arf19 single mutants did not show gravitropic defects, but arf7arf19 double mutant showed abnormal gravitropism in both hypocotyl and roots (Okushima et al., 2005). Furthermore, upon gravistimulation, the asymmetric auxin distribution leads to the expression of $A R F 19$. Mutations within genes that affect activity and stability of auxin carriers also play a role in gravitropism; the axr4 mutant showed gravitropic defects similar to that of auxl mutant. Similar to aux1, the axr4 mutant's gravitropic defect was rescued by application of naphthalene-acetic acid (NAA) but not by IAA or 2,4-dichlorophenoxy-acetic acid (2,4-D) (Masson et al., 2002). Later on, it was confirmed that AXR4, a protein present in endoplasmic reticulum, regulates localization of AUX1 and the agravitropic phenotype of axr4 is caused due to defective AUX1 trafficking in the root epidermis (Dharmasiri et al., 2006). A small secretory peptide in Arabidopsis named GOLVEN (GLV) affects auxin transport by regulating distribution of PIN2 proteins (Whitford et al., 2012). TIR2, which encodes for TRYPTOPHAN AMINOTRANSFERASE a key enzyme in auxin biosynthesis, is also found to be differentially expressed on the lower sides of the roots upon gravistimulation (Yamada et al., 2009). The tir2-1 mutant shows reduced gravitropism upon gravity reorientation (Yamada et al., 2009). In a recent study the early phase of root gravitropism involving auxin-induced ion signaling is regulated by CYCLIC NUCLEOTIDE-GATED CHANNEL 14 (CNGC14) which regulates rapid $\mathrm{Ca}^{2+}$ and $\mathrm{pH}$ changes in response to auxin treatment (Shih et al., 2015). Mutation in this gene leads to reduced auxin mediated gravitropic bending giving us a glimpse of evolutionary conserved auxin machinery which utilizes this module to accelerate gravitropic response (Shih et al., 2015). Further, an auxin-signaling mutant patatin-related phospholipase-AI-1 (pplaI-1) showed increased root coiling response when grown on a non-penetretable agar medium tilted at $45^{\circ}$ suggesting a role for auxin signaling in other root directional response such as root waving and coiling in Arabidopsis (Perrineau et al., 2016). In coming years, more investigation at genetic level will help clear the picture of role of auxin and its significance in controlling root gravitropism.

\section{Ethylene and Its Role in Root Gravitropism}

Besides auxin, ethylene is another phytohormone that has been widely investigated during regulation of gravitropism. Although ethylene is intimately involved in regulating growth at the cellular level, its influence on graviresponses might not be a direct one. Ethylene activates local auxin signaling pathway and regulates root growth by regulating auxin biosynthesis or by modulating the auxin transport (Růzicka et al., 2007). Exogenously applied ethylene could strongly inhibit elongation and curvature of gravistimulated roots. Ethylene also delayed the progression of asymmetric auxin distribution across the root upon gravity-stimulation (Buer et al., 2006). In addition, inhibition of ethylene synthesis or action also reduced gravicurvature in maize roots (Lee and Evans, 1990). The ethylene induced inhibition of gravitropism is not seen in ethylene response1 (etr1) and ethylene insensitive2 (ein2) mutants, this shows that an intact ethylene signaling is required for differential growth upon gravi-stimulation (Buer et al., 2006). Ethylene interacts with auxin to regulate root gravitropism. Mutations in the auxin influx protein AUX1 and in the auxin efflux carrier PIN2/ETHYLENE INSENSITIVE ROOT 1 (EIR1)/WAVY ROOTS6 (WAV6)/AGRAVITROPIC ROOT1 (AGR1) can cause insensitivity to ethylene as well (Pickett et al., 1990; Luschnig et al., 1998). Thus, ethylene insensitivity in the roots of aux 1 and agr1/eir1/pin2/wav6 mutant plants may relate to the role of ethylene in regulating auxin transport and further pointing to the importance of the interactions between these two plant hormone signaling pathways. Similarly, roots of the chirality and gravitropism ( $\operatorname{clg} 1)$ mutant that were resistant to various auxins, also displayed a notable resistance towards ethylene (Ferrari et al., 2000). Ethylene could also suppress the formation of root loops, a gravity dependent growth 
response, in presence of high nutrient and Suc availability (Buer et al., 2003). The roots of 1-aminocyclopropane-1-carboxylic acid-related long hypocotyl 1 (alh1) mutant of Arabidopsis which was less sensitive to ethylene and auxin, displayed a faster response to gravity (Vandenbussche et al., 2003). In tomato, ethylene and auxin crosstalk has been shown to regulate root penetration into the soil (Santisree et al., 2011). The Arabidopsis mutant root handedness 1 (rha1), a heat-shock factor, is resistant to ethylene and is perturbed in roots slanting, gravitropism and auxin physiology (Fortunati et al., 2008). It is also found that ethylene and gravity can also affect root skewing and waving in Arabidopsis (Oliva and Dunand, 2007). Additional evidence suggests that ethylene may be involved in the gravitropic response of roots via its effect on starch metabolism. Thus, exogenous ethylene reduced starch levels in Arabidopsis root columella cells and also the magnitude of gravitropic curvature (Guisinger and Kiss, 1999). Since amyloplasts are believed to be necessary for gravity sensing, these results may imply that ethylene accumulation can modify the gravity perception events. Ethylene biosynthesis enzyme; 1-aminocyclopropane-1-carboxylate (ACC) synthase (ACS) has also been shown to play a role in root gravitropism (Huang et al., 2013). The acs7-1 loss of function mutant showed less sensitivity to the inhibition of root gravitropism by calcium chelator ethylene glycol tetra acetic acid (EGTA) (Huang et al., 2013). The acs7-1 mutant was also less sensitive to inhibition of the gravity response by NPA which could be restored by application of ACC (Huang et al., 2013). Many plant organs show a transient burst of ethylene production when transferred from a vertical to a horizontal position. Altogether, plants have developed the ethylene pathway as a possible mode for coping with environment beneath and above the soil by helping the seedlings to modulate their differential growth. Based on previous and recent findings it is clear that ethylene plays a crucial role in gravitropic growth.

\section{Gibberellins and Its Role in Root Gravitropism}

Gibberellins are prime regulators of cell elongation (Feng et al., 2008), therefore it is reasonable to speculate their involvement in gravistimulation responses. The GA signaling acts through GA-induced proteasomal degradation of repressor proteins of the DELLA family, thereby activating transcription of growth-promoting genes (Schwechheimer, 2008). GAs modulate gravitropism at least partly through transcriptional regulation of IAA19/MSG2 also the agravitropism of msg2-1 mutant was alleviated by inhibiting GA biosynthesis using paclobutrazol (PAC) (Gallego-Bartolomé et al., 2011). In a recent study, it was found that there is an asymmetric distribution of GA during root gravitropic growth (Löfke et al., 2013). GA also stabilizes PIN proteins by preventing their trafficking to the lytic vacuole, thereby stabilizing PIN2 at the lower side of the gravistimulated root. This promotes asymmetric auxin flow and distribution and gravitropic bending (Löfke et al., 2013). These findings provide an emerging picture for GAs to be a part of the complex network in regulating gravitropism and also strengthening auxin action during gravitropic responses.

\section{Abscisic Acid and Its Role in Root Gravitropism}

Abscisic acid exerts mainly inhibitory effects on growth and development. Initial studies on the role of ABA in gravitropism were a little discouraging due to several reasons. Exogenously applied ABA does not inhibit rather promotes root growth, and the inhibitory effect is gained only at concentrations significantly higher than those thought to naturally occur. Also, roots of ABA-deficient plants obtained either by chemically inhibiting ABA synthesis or by specific mutations showed no altered response to gravity (Moore, 1990). When half of the root cap of vertically oriented maize roots was substituted with agar block containing ABA, it had little or no effect on curvature relative to that of controls having plain agar block (Lee et al., 1990). Another study for identification of endogenous growth regulators in graviresponding plant organ found no significant lateral asymmetry of endogenous ABA in root tips of Zea mays and Vicia faba (Mertens and Weiler, 1983). Since ABA plays a positive role in hydrotropism, its putative role in gravitropism is masked (Takahashi et al., 2002). Based on studies with various auxin and ABA mutants, it was hypothesized that ABA may serve as a regulator of auxin transport in root hydrotropic response and a similar interaction may also exist during root gravitropism (Eapen et al., 2005). Recently it was reported that exogenous ABA affects gravitropism by playing a role opposite to that of auxin and it acts as a negative regulator of the root gravitropic response in Arabidopsis (Han et al., 2009). ABA signaling in guard cell involves $\mathrm{K}^{+}$efflux to regulate turgidity which in turn involves Phospholipase D (PLD) (Jacob et al., 1999). It was found that ectopic overexpression of PHOSPHOLIPASE D $\zeta 2$ gene $(P L D \zeta 2)$ enhances root gravitropism in Arabidopsis ( $\mathrm{Li}$ and Xue, 2007). Similarly, ABA treatment and the pld 2 mutation together did not cause an additive effect with each other on root gravitropism suggesting that $P L D \zeta 2$ is involved in ABA signaling that suppresses root gravitropism (Taniguchi et al., 2010). In conclusion, combining these reports on role of $\mathrm{ABA}$ in root gravitropism and -hydrotropism as well as its interactions with auxin, we may imply a probable role for ABA in mediating gravitropism-related responses.

\section{CYTOKININS AND ITS ROLE IN ROOT GRAVITROPISM}

Cytokinins are hormones that regulate cell division and development and play essential and crucial roles in various aspects of plant growth (El-Showk et al., 2013). Several studies have provided evidence for involvement of cytokinin in the gravitropic response. Since cytokinin has a negative regulatory role in root growth, it was suspected that it might also function as an inhibitor of tropic root elongation during gravity response. In roots, cytokinin is produced in the root cap cells, which regulate growth and gravitropism (Aloni et al., 2004). Lateral exogenous application of cytokinin through agar block to a vertically 
growing root induced bending towards the site of application (Aloni et al., 2004). These findings confirmed the inhibitory effect of cytokinin on root elongation during gravitropic bending (Aloni et al., 2004). When vertically growing Arabidopsis roots were gravistimulated, free cytokinin was radially transported and got asymmetrically distributed to the lower side of root cap within 30 min as detected by ARR5::GUS expression (Aloni et al., 2004).

Cytokinin may also interact with auxin machineries to regulate gravitropic response (Philosoph-Hadas et al., 2005). The cytokinin induced root curling 1 ( $c k r c 1)$ mutant of Arabidopsis which is allelic to taal exhibits defective root gravitropic response which was rescued by exogenous application of auxin and increased resistance to cytokinin in primary root growth (Zhou et al., 2011). Depletion of endogenous cytokinins by overexpressing CYTOKININ OXIDASE DEHYDROGENASE $(C K X)$ genes caused lateral expansion of the auxin maxima, i.e., from columella to lateral root cap (Pernisová et al., 2009). Further, cytokinin signaling defective mutant ahk3 and cytokinin deficient Pro35S:AtCKX transgenic lines showed defects in both auxin redistribution as well as root gravitropic response (Pernisova et al., 2016). Upon gravistimulation, the AtCKX3 overexpression and ahk3 mutant lines showed WT like pattern for PIN3 and PIN7 relocalization in columella while the pin3pin4pin7 triple mutant did not show any defect in cytokinin sensitivity. On the other hand, depleting endogenous cytokinins via AtCKX overexpression caused alteration in cellular distribution of auxin influx carrier AUX1 suggesting that cytokinin largely targets AUX1-mediated auxin transport rather than PIN-mediated auxin transport to affect root gravitropism (Pernisova et al., 2016). In an interesting finding, role of cytokinin was established in cytokinin-induced root tip reorientation growth response which involves members of two-component system, i.e., ARABIDOPSIS HISTIDINE KINASEs (AHKs); and type-A and type-B RESPONSE REGULATORs (ARRs) (Kushwah et al., 2011). In conclusion, cytokinin seems to play a key regulatory role in root gravitropism. However, a close and complex interaction between cytokinin and auxin together with other hormones seems to take place in regulation of gravitropic growth.

\section{Brassinosteroid (BR) and Its Role in Root Gravitropism}

Brassinosteroid (BRs) are regarded to be essential substances for growth and development in plants, and their occurrence has been demonstrated in all plant organs. Brassinolide (BL) was found to increase the gravitropic response of roots by increasing their sensitivity to IAA (Kim et al., 2000). BL can also stimulate the gravitropic response in maize roots via both ethylene dependent as well as independent mechanisms (Chang et al., 2004). In Pisum sativum roots, 24-epibrassinolide (EBL) treatment could reduce the average lag-time needed for gravitropic response initiation in a dose dependent manner (Amzallag and Vaisman, 2006). Further, treatment with steroid biosynthesis inhibitor clotrimazole could prevent the gravitropic response initiation which was partly rescued by EBL application (Amzallag and Vaisman, 2006). Exogenous application of BR promotes acropetal and basipetal auxin transport in Arabidopsis and Brassica roots ( $\mathrm{Li}$ et al., 2005). BRs can also regulate gravitropic response of Arabidopsis roots by stimulating the activity of ROP2 GTPase, which mediates polar auxin transport, resulting in an increased gravitropic response (Li et al., 2005). However, both BL as well as IAA, at higher concentrations, antagonize the gravitropic curvature response induced by each other via modulating biosynthesis of the counterpart hormone (Kim et al., 2007). Like auxin, exogenous BR application could also affect actin cytoskeleton configuration and PIN2 localization patterns which in turn modulates auxin gradients maintenance and affect gravitropic responses in Arabidopsis roots (Lanza et al., 2012). These results show that BRs have a prominent role in controlling root gravitropic responses.

\section{Jasmonic Acid and Its Role in Root Gravitropism}

Jasmonic acid is mostly studied in regulating plant defense but its function during plant growth and development is also fast emerging. There is very less information available in context of root gravitropism. As modulation of JA homeostasis as well as signal transduction can mimic auxin effects on root development, we assume it to have some effect on root gravitropic responses as well. In rice coleoptiles, the total content of JA is found to be increased upon gravity reorientation (Gutjahr et al., 2005). Also, a JA gradient is established opposite to the internal auxin gradient across the stimulated organ positively modulating gravitropic curvature. This JA-gradient in response to gravitropic stimulation is developed in an IAA-independent manner (Gutjahr et al., 2005). Interestingly, a JA-deficiency in rice mutant hebiba could not abolish its gravitropic response suggesting that JA might not be essential but could accelerate the gravitropic bending response (Gutjahr et al., 2005). In Arabidopsis, tryptophan conjugates of JA (JA-Trp) which act as IAA-antagonists can cause root agravitropism in a dose-dependent manner. The JATrp functions in a TIR1-dependent manner but are independent of COI1 (Staswick, 2009). In conclusion, JA might regulate root gravitropic responses via affecting auxin biosynthesis and gradient formation via modulating polar auxin distribution.

\section{LIGHT AS A TRIGGER FOR CHANGE IN ROOT GRAVITROPISM}

Light is an essential component for energy production and survival in plants. Light regulates nearly all stages of plant development on the basis of its quantity, quality and directionality. On the other hand, gravity is a constant stimulus providing plants with the critical information about its surroundings and thus guiding plant growth. Evidences have shown that light is required for triggering gravitropism in plants. The roots of Vanilla planifolia when exposed to light responded rapidly to the stimulus of gravity (Irvine and Freyre, 1961). In Convolvulus arvensis root when exposed to light showed positive orthogeotropic response. Red light enhanced the response and far-red-light exposure reversed the effect of red light (Tepfer and Bonnett, 1972). Different light spectrum 
is sensed by a variety of photoreceptors such as phytochromes, cryptochromes, phototropins, zeitlupes, and UVR8 (de Wit et al., 2016). There are few evidences in literature involving light mediated regulation of root movements. Roots also possess the phytochromes which sense $\mathrm{R}$ and FR light to mediate root elongation and directional growth (Correll and Kiss, 2005). The phyB and $p h y A B$ mutants showed delayed response toward gravity reorientation (Correll and Kiss, 2005). Phytochrome-A has been shown to play distinct role in red light-induced positive root phototropism (Kiss et al., 2003). Phytochrome-A also inhibits blue light mediated negative phototropism in Arabidopsis roots (Kiss et al., 2007). Light can also control root orthogravitropic response in maize via $\mathrm{Ca}^{2+} /$ calmodulindependent protein kinase cascade (Lu and Feldman, 1997). Some evidence also suggests that these phytochrome also alters jasmonic acid response and sensitivity in roots (Costigan et al., 2011). In Arabidopsis, blue light photoreceptor PHOT1 is highly expressed in the root tissue (Mo et al., 2015). PHOT1 regulates the expression of PINOID kinase and PP2A phosphatase to mediate PIN3 polarization in the root thereby facilitating root bending (Zhang et al., 2013). Interestingly, PHOT1 is also found to regulate PIN2 localization through NPH3 proteins and blue light illumination was able to reduce gravitropic bending (Wan et al., 2012). Under microgravity conditions, blue light induced phototropism in roots was greatly reduced at $0.1 \mathrm{~g}$ and masked at $0.3 \mathrm{~g}$ and higher gravity levels displaying a novel response (Vandenbrink et al., 2016). PINOID kinases which are known to regulate auxin efflux carriers have been shown to negatively regulate root phototropism (Haga and Sakai, 2015). When Arabidopsis roots are illuminated with unilateral blue-light PID proteins are accumulated on the non-illuminated side of the root (Haga and Sakai, 2015). Flavonols in the transition zone have been shown to function as a positional signal interacting with hormonal and ROS pathways to regulate root directional growth to light (Silva-Navas et al., 2016). Upon root illumination, the flavonols accumulate towards the side exposed to light and causing differential growth promoting root movement away from light (Silva-Navas et al., 2016). The possible role of light in root gravitropism can further be strengthened by the fact that shoot phytochrome-B induces and stabilizes the expression of ELONGATED HYPOCOTYL5 (HY5) protein which as a shoot-to-root mobile signal regulates root growth and nitrate uptake (Chen et al., 2016). Later micrografting studies using Col-0 and phyB-9 plants followed by subsequent illumination of grafted shoots suggested that root derived phyB regulates root gene expression (Lee et al., 2016). Since photoreceptors have a high sensitivity towards different lights, it is possible that a small level of stem piped light can bring significant changes in root architecture. It is now proven that not only light in the range of Far-Red and near Infra-Red is transmitted through the stems to roots but also light in the range of green to red is also effectively transmitted as a signal (Chen et al., 2016; Lee et al., 2016). Plants adapt to the changing environment by sensing the intensity and quality of light to regulate root growth and development and root gravitropism (Lee et al., 2016). Increased light fluxes can also modulate the levels of photosynthetically generated sugars that in turn can affect the directional movements in Arabidopsis such as root coiling, waving and deviation from vertical gravity vector (Singh et al., 2014a). In the event of recent developments, further identification of light induced mobile signals will help in finding new targets to modulate root gravitropism and architecture. A detailed analysis of vascular tissues and how they transduce light signals will be helpful in regulating plant architecture.

\section{GLUCOSE AS AN EMERGING PLAYER IN ROOT GRAVITROPISM}

Nutrient availability is a major factor controlling growth in a constantly changing environment. Plants, like other living organisms, need to maintain nutrient and energy homeostasis within cells and tissues for growth. They fulfil their energy requirement by fixing light into a metabolizable form via photosynthesis where carbohydrate (sugar) energy is utilized as fuel for growth and/or stored as reserve. Sugars are the prime carbon and energy source to build and fuel cells, and also acquired important regulatory functions in controlling metabolism, stress resistance, growth and development. Sugars also have an important signaling function and act like hormones in translating nutrient status to regulate growth and floral transition (Koch, 2004; Price et al., 2004; Rolland et al., 2006; Ramon et al., 2008; Smeekens et al., 2010; Lastdrager et al., 2014). Sucrose being the primary transport sugar, can be sensed as a signal either directly (Chiou and Bush, 1998); or via its hexose cleavage products such as glucose (Glc), UDPglucose and fructose (Rolland et al., 2002; Price et al., 2004; Li et al., 2011). Glc being the second most abundant sugar in Arabidopsis has an important role to play in plant growth and development (Moore et al., 2003). Transcriptomics as well as genetic analyses have revealed that, in plants, many genes are regulated by Glc and its signaling networks (Price et al., 2004; Li et al., 2006). In recent years, a pivotal role of Glc in plant growth and development and key players in the Glc signaling network have been uncovered using Arabidopsis as the prime model system (Koch, 1996; Smeekens, 2000; Rolland et al., 2002; León and Sheen, 2003; Gibson, 2005; Rolland et al., 2006; Smith and Stitt, 2007). In plants, several sugar sensors exist which regulate growth and development. These sensors include HEXOKINASE 1 (HXK1) (Moore et al., 2003), REGULATOR OF G-PROTEIN SIGNALING 1 (RGS1) (Chen et al., 2003) and other sensors which respond to different sugars or sugarderived metabolites, including trehalose-6-phosphate (Tre6P) and fructose etc. Thus, three distinct Glc signal transduction pathways operate in plants. One is the HXK1-dependent pathway governed by AtHXK1-mediated signaling function. The second is a HXK1-independent pathway where changes in gene expression are modulated by Glc but is independent of AtHXK1. The third is a glycolysis-dependent pathway that utilizes SUCROSE NON-FERMENTING RELATED KINASE1 (SnRK1)/TARGET OF RAPAMYCIN (TOR) pathway (Fu et al., 2014).

Sugar signaling pathway exhibits crosstalk with other response pathways such as those involved in light, phytohormones and stress responses. In plants, sugar and phytohormone signal cross-talks have been shown to modulate critical growth 
and developmental processes such as embryo establishment, seed germination, and early seedling growth and development (Gazzarrini and McCourt, 2001; Rolland et al., 2002; León and Sheen, 2003; Gibson, 2004, 2005; Rolland et al., 2006; Mishra et al., 2009; Kushwah et al., 2011; Gupta et al., 2012; Singh et al., 2014a,b; Gupta et al., 2015a,b). Apart from regulation of plant growth and development in general, there are few instances where sugar signals are reported to be controlling various directional growth responses in plants either independently or via interaction with other signals. For example, in maize, Glc from kernel to shoot becomes asymmetrically distributed in cortical tissue upon gravity-stimulation similar to radiolabelled IAA. This asymmetric distribution of Glc could also involve a lateral transport system as described for auxin (Momonoki, 1988). Glc- and Auxin signaling has also been found to interact extensively in regulating root gravitropism in Arabidopsis. The primary roots of Arabidopsis seedlings displayed a significant deviation from their vertical growth direction upon exogenous application of higher Glc concentrations (Mishra et al., 2009; Singh et al., 2014a). Also, the root gravitropic bending kinetics was significantly delayed in seedlings treated with high concentrations of Glc. Glc altered the root gravitropic response via both HXK1-dependent as well as HXK1-independent mechanisms (Singh et al., 2014b). Multiple phytohormone signaling components such as BR, cytokinin, ethylene and auxin work downstream to Glc to cause root deviation from vertical gravity vector response (Singh et al., 2014a,b). Glc promotes BRI1 mediated signaling by inhibiting the activity of Protein Phosphatase 2A (PP2A) (Singh et al., 2014b). Cytokinin and ethylene signals work further downstream and could antagonize this Glc response (Singh et al., 2014a). Exogenous Glc was able to alter rate of polar auxin transport and might utilize auxin transport machinery and further downstream components of auxin response pathway to modulate root gravitropic responses (Mishra et al., 2009; Singh et al., 2014b). Glc could also affect coiling and waving responses in Arabidopsis seedling root (Singh et al., 2014b). Glc interact synergistically with cytokinin to execute a novel root tip directional growth response in light grown Arabidopsis seedling (Kushwah et al., 2011). In Arabidopsis, TOR kinase dependent sugar/Glc signaling and energy homeostasis could also regulate root growth and development (Xiong et al., 2013). TOR-dependent signaling may interfere with auxin signaling to regulate root gravitropic response. The TOR RNAi seedlings were found to be defective in gravitropic bending response (Schepetilnikov et al., 2013). Also, chemical inhibition of TOR complex activity via Torin1 pre-treatment could abolish the gravitropic bending response in Arabidopsis roots (Schepetilnikov et al., 2013). Rapamycin inhibits TOR kinase activity via FK506-binding protein 12 (FKBP12). Transgenic plants having functional FKBP12 in DR5::GUS background (DR5/BP12) showed loss of gravitropism upon treatment with rapamycin and another inhibitor of TOR, KU63794 (Deng et al., 2016). Recently, possible roles for TOR signaling complex in dictating various other directional growth responses of roots such as light escape in soil and salt avoidance have also been described (Yokawa and Baluška, 2015). These reports together reveal that a complex signaling network between sugar and plant hormones especially auxin response, light signals and energy balance homeostasis in cells of root apices operate to optimized directional growth behaviors in plant roots.

\section{CONCLUSION}

Plants, being sessile organisms, use the coordinated action of several signaling pathways to grow and develop optimally in response to a changing environment. We know that light is an important factor in determining the directionality of plant growth. But gravity, a force that causes objects to fall and holds the planets in their orbits around the sun, is also critically important. Root directional growth and growth angle determines the area coverage in which it can capture water and nutrients and guides a plant to utilize nutrients that are unevenly distributed in soil. Plants have evolved to respond to different stimuli to help them orient to their best advantage. The growth and development of plants is mainly dependent on the platform set by the integrations of various signals such as light, gravity, nutrient, phytohormones etc. There are numerous examples of synergy, antagonism, and causal relationships among the different signaling pathways under various molecular and physiological processes, such as the control of cell expansion and divisions that define the architecture of vascular plants. Gravitropism is one of the major factors that determine root growth direction. Mechanism and control of gravi-response is a highly complex process which also involves several growth regulators. Recently introduced novel fluorescent $\mathrm{pH}$ indicator 8-hydroxypyrene-1,3,6-trisulfonic acid trisodium salt (HPTS) have enabled better understanding of asymmetric apoplast alkalization in gravistimulated roots and subsequent processes (Barbez et al., 2017). The identification of negative gravitropic response of roots (ngr) mutants in medicago and Arabidopsis, have provided further insight into the mechanism of root gravitropism (Ge and Chen, 2016). The loss of function of NGR genes (AtNGR1; At1g17400, AtNGR2; At1g72490 and AtNGR3; At1g19115) reverses the root growth direction and the roots start to grow upwards against gravity vector (Ge and Chen, 2016). Further functional characterization of these mutants will help in connecting the dots of the model of gravitropism. New imaging techniques with rotating stages and tracking software such as Tip-Tracker has efficiently been used for imaging root growth and shown promises for the future of root gravitropic studies (von Wangenheim et al., 2017). Overall scenario suggests that in the phytohormonal hierarchy of gravitropic response, auxin homeostasis and cellular dynamics forms the converging point for most of the signals. However, individual roles of other phytohormones cannot be denied and it is the need of the hour to investigate the critical connecting links between these signals to strengthen our understanding towards the root tropic movements. With the availability of a plethora of mutants in Arabidopsis as well as other crop models and advanced molecular genetic studies, these complex interactions in various signaling pathways can be modulated to develop a range of improved plant varieties with optimal root growth behavior adapted towards a range of environmental challenges. 


\section{AUTHOR CONTRIBUTIONS}

All authors have made intellectual contribution to the article, and approved it for publication. MS, AG, and AL conceptualized the article. MS and AG wrote the article and did final editing.

\section{REFERENCES}

Aloni, R., Langhans, M., Aloni, E., and Ullrich, C. I. (2004). Role of cytokinin in the regulation of root gravitropism. Planta 220, 177-182. doi: 10.1007/s00425-0041381-8

Amzallag, G., and Vaisman, J. (2006). Influence of brassinosteroids on initiation of the root gravitropic response in Pisum sativum seedlings. Biol. Plant. 50, 283-286. doi: 10.1007/s10535-006-0021-5

Baldwin, K. L., Strohm, A. K., and Masson, P. H. (2013). Gravity sensing and signal transduction in vascular plant primary roots. Am. J. Bot. 100, 126-142. doi: 10.3732/ajb.1200318

Band, L. R., Wells, D. M., Larrieu, A., Sun, J., Middleton, A. M., French, A. P., et al. (2012). Root gravitropism is regulated by a transient lateral auxin gradient controlled by a tipping-point mechanism. Proc. Natl. Acad. Sci. U.S.A. 109, 4668-4673. doi: 10.1073/pnas.1201498109

Barbez, E., Dünser, K., Gaidora, A., Lendl, T., and Busch, W. (2017). Auxin steers root cell expansion via apoplastic $\mathrm{pH}$ regulation in Arabidopsis thaliana. Proc. Natl. Acad. Sci. U.S.A. 114, E4884-E4893. doi: 10.1073/pnas.1613499114

Baster, P., Robert, S., Kleine-Vehn, J., Vanneste, S., Kania, U., Grunewald, W., et al. (2013). SCF(TIR1/AFB)-auxin signalling regulates PIN vacuolar trafficking and auxin fluxes during root gravitropism. EMBO J. 32, 260-274. doi: 10.1038/ emboj.2012.310

Blancaflor, E., Hou, G.-C., and Mohamalawari, D. (2003). The promotive effect of latrunculin B on maize root gravitropism is concentration dependent. $A d v$. Space Res. 31, 2215-2220. doi: 10.1016/S0273-1177(03)00247-3

Blancaflor, E. B. (2013). Regulation of plant gravity sensing and signaling by the actin cytoskeleton. Am. J. Bot. 100, 143-152. doi: 10.3732/ajb.1200283

Blancaflor, E. B., Fasano, J. M., and Gilroy, S. (1998). Mapping the functional roles of cap cells in the response of Arabidopsis primary roots to gravity. Plant Physiol. 116, 213-222. doi: 10.1104/pp.116.1.213

Boonsirichai, K., Sedbrook, J. C., Chen, R., Gilroy, S., and Masson, P. H. (2003). ALTERED RESPONSE TO GRAVITY is a peripheral membrane protein that modulates gravity-induced cytoplasmic alkalinization and lateral auxin transport in plant statocytes. Plant Cell 15, 2612-2625. doi: 10.1105/tpc.015560

Braun, M., and Limbach, C. (2006). Rhizoids and protonemata of characean algae: model cells for research on polarized growth and plant gravity sensing. Protoplasma 229, 133-142. doi: 10.1007/s00709-006-0208-9

Buer, C. S., Masle, J., and Wasteneys, G. O. (2000). Growth conditions modulate root-wave phenotypes in Arabidopsis. Plant Cell Physiol. 41, 1164-1170. doi: $10.1093 / \mathrm{pcp} / \mathrm{pcd} 042$

Buer, C. S., Sukumar, P., and Muday, G. K. (2006). Ethylene modulates flavonoid accumulation and gravitropic responses in roots of Arabidopsis. Plant Physiol. 140, 1384-1396. doi: 10.1104/pp.105.075671

Buer, C. S., Wasteneys, G. O., and Masle, J. (2003). Ethylene modulates root-wave responses in Arabidopsis. Plant Physiol. 132, 1085-1096. doi: 10.1104/pp.102. 019182

Chang, S. C., Kim, Y. S., Lee, J. Y., Kaufman, P. B., Kirakosyan, A., Yun, H. S., et al. (2004). Brassinolide interacts with auxin and ethylene in the root gravitropic response of maize (Zea mays). Physiol. Plant. 121, 666-673. doi: 10.1111/j.00319317.2004.00356.x

Chen, J.-G., Willard, F. S., Huang, J., Liang, J., Chasse, S. A., Jones, A. M., et al. (2003). A seven-transmembrane RGS protein that modulates plant cell proliferation. Science 301, 1728-1731. doi: 10.1126/science. 1087790

Chen, X., Yao, Q., Gao, X., Jiang, C., Harberd, N. P., and Fu, X. (2016). Shoot-toroot mobile transcription factor HY5 coordinates plant carbon and nitrogen acquisition. Curr. Biol. 26, 640-646. doi: 10.1016/j.cub.2015.12.066

Chiou, T. J., and Bush, D. R. (1998). Sucrose is a signal molecule in assimilate partitioning. Proc. Natl. Acad. Sci. U.S.A. 95, 4784-4788. doi: 10.1073/pnas.95. 8.4784

\section{ACKNOWLEDGMENT}

The authors are thankful to Department of Science and Technology, Government of India for financial support (BT/PR3302/AG $\mathrm{R} / 02 / 814 / 2011$ ) and research fellowships to MS (DST/INSPIRE/ 04/2016/000634) and AG (DST/INSPIRE/04/2015/001952).

Collings, D. A., Zsuppan, G., Allen, N. S., and Blancaflor, E. B. (2001). Demonstration of prominent actin filaments in the root columella. Planta 212, 392-403. doi: 10.1007/s004250000406

Correll, M. J., and Kiss, J. Z. (2005). The roles of phytochromes in elongation and gravitropism of roots. Plant Cell Physiol. 46, 317-323. doi: 10.1093/pcp/pci038

Costigan, S. E., Warnasooriya, S. N., Humphries, B. A., and Montgomery, B. L. (2011). Root-localized phytochrome chromophore synthesis is required for photoregulation of root elongation and impacts root sensitivity to jasmonic acid in Arabidopsis. Plant Physiol. 157, 1138-1150. doi: 10.1104/pp.111.184689

Dalal, J., Lewis, D. R., Tietz, O., Brown, E. M., Brown, C. S., Palme, K., et al. (2016). ROSY1, a novel regulator of gravitropic response is a stigmasterol binding protein. J. Plant Physiol. 196, 28-40. doi: 10.1016/j.jplph.2016.03.011

Darwin, C., and Darwin, F. (1880). The Power of Movement in Plants. $\iota$ London: John Murray. doi: 10.5962/bhl.title.102319

de Wit, M., Galvão, V. C., and Fankhauser, C. (2016). Light-mediated hormonal regulation of plant growth and development. Annu. Rev. Plant Biol. 67, 513-537. doi: 10.1146/annurev-arplant-043015-112252

Deng, K., Yu, L., Zheng, X., Zhang, K., Wang, W., Dong, P., et al. (2016). Target of rapamycin is a key player for auxin signaling transduction in Arabidopsis. Front. Plant Sci. 7:291. doi: 10.3389/fpls.2016.00291

Dharmasiri, S., Swarup, R., Mockaitis, K., Dharmasiri, N., Singh, S. K., Kowalchyk, M., et al. (2006). AXR4 is required for localization of the auxin influx facilitator AUX1. Science 312, 1218-1220. doi: 10.1126/science.1122847

Digby, J., and Firn, R. D. (1995). The gravitropic set-point angle (GSA): the identification of an important developmentally controlled variable governing plant architecture. Plant Cell Environ. 18, 1434-1440. doi: 10.1111/j.1365-3040. 1995.tb00205.x

Eapen, D., Barroso, M. L., Ponce, G., Campos, M. E., and Cassab, G. I. (2005). Hydrotropism: root growth responses to water. Trends Plant Sci. 10, 44-50. doi: 10.1016/j.tplants.2004.11.004

El-Showk, S., Ruonala, R., and Helariutta, Y. (2013). Crossing paths: cytokinin signalling and crosstalk. Development 140, 1373-1383. doi: 10.1242/dev.086371

Fasano, J. M., Swanson, S. J., Blancaflor, E. B., Dowd, P. E., Kao, T. H., and Gilroy, S. (2001). Changes in root cap $\mathrm{pH}$ are required for the gravity response of the Arabidopsis root. Plant Cell 13, 907-921. doi: 10.1105/tpc.13.4.907

Feng, S., Martinez, C., Gusmaroli, G., Wang, Y., Zhou, J., Wang, F., et al. (2008). Coordinated regulation of Arabidopsis thaliana development by light and gibberellins. Nature 451, 475-479. doi: 10.1038/nature 06448

Ferrari, S., Piconese, S., Tronelli, G., and Migliaccio, F. (2000). A new Arabidopsis thaliana root gravitropism and chirality mutant. Plant Sci. 158, 77-85. doi: 10.1016/S0168-9452(00)00309-5

Fitzelle, K. J., and Kiss, J. Z. (2001). Restoration of gravitropic sensitivity in starchdeficient mutants of Arabidopsis by hypergravity. J. Exp. Bot. 52, 265-275. doi: 10.1093/jexbot/52.355.265

Fortunati, A., Piconese, S., Tassone, P., Ferrari, S., and Migliaccio, F. (2008). A new mutant of Arabidopsis disturbed in its roots, right-handed slanting, and gravitropism defines a gene that encodes a heat-shock factor. J. Exp. Bot. 59, 1363-1374. doi: 10.1093/jxb/ern047

Friml, J. (2003). Auxin transport-shaping the plant. Curr. Opin. Plant Biol. 6, 7-12. doi: 10.1016/S1369526602000031

Friml, J., Wiśniewska, J., Benková, E., Mendgen, K., and Palme, K. (2002). Lateral relocation of auxin efflux regulator PIN3 mediates tropism in Arabidopsis. Nature 415, 806-809. doi: 10.1038/415806a

Fu, Y., Lim, S., Urano, D., Tunc-Ozdemir, M., Phan, N. G., Elston, T. C., et al. (2014). Reciprocal encoding of signal intensity and duration in a glucosesensing circuit. Cell 156, 1084-1095. doi: 10.1016/j.cell.2014.01.013

Fukaki, H., Tameda, S., Masuda, H., and Tasaka, M. (2002). Lateral root formation is blocked by a gain-of-function mutation in the SOLITARY-ROOT/IAA14 
gene of Arabidopsis. Plant J. 29, 153-168. doi: 10.1046/j.0960-7412.2001. 01201.x

Gallego-Bartolomé, J., Kami, C., Fankhauser, C., Alabadí, D., and Blázquez, M. A. (2011). A hormonal regulatory module that provides flexibility to tropic responses. Plant Physiol. 156, 1819-1825. doi: 10.1104/pp.111.173971

Gazzarrini, S., and McCourt, P. (2001). Genetic interactions between ABA, ethylene and sugar signaling pathways. Curr. Opin. Plant Biol. 4, 387-391. doi: 10.1016/S1369-5266(00)00190-4

Ge, L., and Chen, R. (2016). Negative gravitropism in plant roots. Nat. Plants 2, 16155. doi: 10.1038/nplants.2016.155

Geldner, N., Anders, N., Wolters, H., Keicher, J., Kornberger, W., Muller, P., et al. (2003). The Arabidopsis GNOM ARF-GEF mediates endosomal recycling, auxin transport, and auxin-dependent plant growth. Cell 112, 219-230. doi: 10.1016/S0092-8674(03)00003-5

Gibson, S. I. (2004). Sugar and phytohormone response pathways: navigating a signalling network. J. Exp. Bot. 55, 253-264. doi: 10.1093/jxb/erh048

Gibson, S. I. (2005). Control of plant development and gene expression by sugar signaling. Curr. Opin. Plant Biol. 8, 93-102. doi: 10.1016/j.pbi.2004.11.003

Guisinger, M. M., and Kiss, J. Z. (1999). The influence of microgravity and spaceflight on columella cell ultrastructure in starch-deficient mutants of Arabidopsis. Am. J. Bot. 86, 1357-1366. doi: 10.2307/2656918

Gupta, A., Singh, M., Jones, A. M., and Laxmi, A. (2012). Hypocotyl directional growth in arabidopsis: a complex trait. Plant Physiol. 159, 1463-1476. doi: 10.1104/pp.112.195776

Gupta, A., Singh, M., and Laxmi, A. (2015a). Interaction between glucose and brassinosteroid during the regulation of lateral root development in Arabidopsis. Plant Physiol. 168, 307-320. doi: 10.1104/pp.114.256313

Gupta, A., Singh, M., and Laxmi, A. (2015b). Multiple Interactions between glucose and brassinosteroid signal transduction pathways in Arabidopsis are uncovered by whole-genome transcriptional profiling. Plant Physiol. 168, 1091-1105. doi: 10.1104/pp.15.00495

Gutjahr, C., Riemann, M., Müller, A., Düchting, P., Weiler, E. W., and Nick, P. (2005). Cholodny-went revisited: a role for jasmonate in gravitropism of rice coleoptiles. Planta 222, 575-585. doi: 10.1007/s00425-005-0001-6

Haberlandt, G. (1900). Über die Perception des geotropischen Reizes. Ber. Deut. Bot. Ges. 18, 261-272.

Haga, K., and Sakai, T. (2015). PINOID functions in root phototropism as a negative regulator. Plant Signal. Behav. 10:e998545 doi: 10.1080/15592324.2014. 998545

Han, W., Rong, H., Zhang, H., and Wang, M.-H. (2009). Abscisic acid is a negative regulator of root gravitropism in Arabidopsis thaliana. Biochem. Biophys. Res. Commun. 378, 695-700. doi: 10.1016/j.bbrc.2008.11.080

Harrison, B. R., and Masson, P. H. (2008). ARL2, ARG1 and PIN3 define a gravity signal transduction pathway in root statocytes. Plant J. 53, 380-392. doi: 10.1111/j.1365-313X.2007.03351.x

Hou, G., Kramer, V. L., Wang, Y. S., Chen, R., Perbal, G., Gilroy, S., et al. (2004). The promotion of gravitropism in Arabidopsis roots upon actin disruption is coupled with the extended alkalinization of the columella cytoplasm and a persistent lateral auxin gradient. Plant J. 39, 113-125. doi: 10.1111/j.1365-313X. 2004.02114.x

Huang, S.-J., Chang, C.-L., Wang, P.-H., Tsai, M.-C., Hsu, P.-H., and Chang, I.F. (2013). A type III ACC synthase, ACS7, is involved in root gravitropism in Arabidopsis thaliana. J. Exp. Bot. 64, 4343-4360. doi: 10.1093/jxb/ert241

Irvine, J. E., and Freyre, R. H. (1961). Diageotropism in Vanilla roots. Science 134, 56-57. doi: 10.1126/science.134.3471.56

Ishikawa, H., Natori, E., and Evans, M. (2007). Use of ROTATO/Random Positioning Machine (R/RPM) technology to investigate gravity sensing and the gravitropic motor response of maize roots. Gravit. Space Res. 20, 101-102.

Jacob, T., Ritchie, S., Assmann, S. M., and Gilroy, S. (1999). Abscisic acid signal transduction in guard cells is mediated by phospholipase D activity. Proc. Natl. Acad. Sci. U.S.A. 96, 12192-12197. doi: 10.1073/pnas.96.21.12192

Kim, S. K., Chang, S. C., Lee, E. J., Chung, W. S., Kim, Y. S., Hwang, S., et al. (2000). Involvement of brassinosteroids in the gravitropic response of primary root of maize. Plant Physiol. 123, 997-1004. doi: 10.1104/pp.123.3.997

Kim, T.-W., Lee, S. M., Joo, S.-H., Yun, H. S., Lee, Y. E. W., Kaufman, P. B., et al. (2007). Elongation and gravitropic responses of Arabidopsis roots are regulated by brassinolide and IAA. Plant Cell Environ. 30, 679-689. doi: 10.1111/j.13653040.2007.01659.x
Kiss, J. Z., Correll, M. J., Mullen, J. L., Hangarter, R. P., and Edelmann, R. E. (2007). Root phototropism: how light and gravity interact in shaping plant form. Gravit. Space Res. 16, 55-60.

Kiss, J. Z., Mullen, J. L., Correll, M. J., and Hangarter, R. P. (2003). Phytochromes A and B mediate red-light-induced positive phototropism in roots. Plant Physiol. 131, 1411-1417. doi: 10.1104/pp.013847

Kiss, J. Z., Wright, J. B., and Caspar, T. (1996). Gravitropism in roots of intermediate-starch mutants of Arabidopsis. Physiol. Plant. 97, 237-244.break doi: 10.1034/j.1399-3054.1996.970205.x

Kleine-Vehn, J., Ding, Z., Jones, A. R., Tasaka, M., Morita, M. T., and Friml, J. (2010). Gravity-induced PIN transcytosis for polarization of auxin fluxes in gravity-sensing root cells. Proc. Natl. Acad. Sci. U.S.A. 107, 22344-22349. doi: $10.1073 /$ pnas.1013145107

Koch, K. (2004). Sucrose metabolism: regulatory mechanisms and pivotal roles in sugar sensing and plant development. Curr. Opin. Plant Biol. 7, 235-246. doi: 10.1016/j.pbi.2004.03.014

Koch, K. E. (1996). Carbohydrate-modulated gene expression in plants. Annu. Rev. Plant Physiol. Plant Mol. Biol. 47, 509-540. doi: 10.1146/annurev.arplant. 47.1.509

Kushwah, S., Jones, A. M., and Laxmi, A. (2011). Cytokinin interplay with ethylene, auxin, and glucose signaling controls Arabidopsis seedling root directional growth. Plant Physiol. 156, 1851-1866. doi: 10.1104/pp.111.175794

Kuznetsov, O. A., and Hasenstein, K. H. (1996). Intracellular magnetophoresis of amyloplasts and induction of root curvature. Planta 198, 87-94. doi: 10.1007/ BF00197590

Kuznetsov, O. A., and Hasenstein, K. H. (1997). Magnetophoretic induction of curvature in coleoptiles and hypocotyls. J. Exp. Bot. 48, 1951-1957. doi: $10.1093 / \mathrm{jxb} / 48.11 .1951$

Lanza, M., Garcia-Ponce, B., Castrillo, G., Catarecha, P., Sauer, M., RodriguezSerrano, M., et al. (2012). Role of actin cytoskeleton in brassinosteroid signaling and in its integration with the auxin response in plants. Dev. Cell 22, 1275-1285. doi: 10.1016/j.devcel.2012.04.008

Lastdrager, J., Hanson, J., and Smeekens, S. (2014). Sugar signals and the control of plant growth and development. J. Exp. Bot. 65, 799-807. doi: 10.1093/jxb/ert474

Lee, H.-J., Ha, J.-H., Kim, S.-G., Choi, H.-K., Kim, Z. H., Han, Y.-J., et al. (2016). Stem-piped light activates phytochrome B to trigger light responses in Arabidopsis thaliana roots. Sci. Signal. 9:ra106. doi: 10.1126/scisignal.aaf6530

Lee, J. S., and Evans, M. L. (1990). Effects of ethylene on the kinetics of curvature and auxin redistribution in gravistimulated roots of Zea mays. Plant Physiol. 94, 1770-1775. doi: 10.1104/pp.94.4.1770

Lee, J. S., Hasenstein, K. H., Mulkey, T. J., Yang, R. L., and Evans, M. L. (1990). Effects of abscisic acid and xanthoxin on elongation and gravitropism in primary roots of Zea mays. Plant Sci. 68, 17-26. doi: 10.1016/0168-9452(90) 90147-G

Lee, J. S., Mulkey, T. J., and Evans, M. L. (1983a). Gravity-induced polar transport of calcium across root tips of maize. Plant Physiol. 73, 874-876.

Lee, J. S., Mulkey, T. J., and Evans, M. L. (1983b). Reversible loss of gravitropic sensitivity in maize roots after tip application of calcium chelators. Science 220, 1375-1376.

Lee, J. S., Mulkey, T. J., and Evans, M. L. (1984). Inhibition of polar calcium movement and gravitropism in roots treated with auxin-transport inhibitors. Planta 160, 536-543. doi: 10.1007/BF00411142

Legué, V., Blancaflor, E., Wymer, C., Perbal, G., Fantin, D., and Gilroy, S. (1997). Cytoplasmic free Ca2+ in Arabidopsis roots changes in response to touch but not gravity. Plant Physiol. 114, 789-800. doi: 10.1104/pp.114. 3.789

Leitz, G., Kang, B.-H., Schoenwaelder, M. E. A., and Staehelin, L. A. (2009). Statolith sedimentation kinetics and force transduction to the cortical endoplasmic reticulum in gravity-sensing Arabidopsis columella cells. Plant Cell 21, 843-860. doi: 10.1105/tpc.108.065052

León, P., and Sheen, J. (2003). Sugar and hormone connections. Trends Plant Sci. 8, 110-116. doi: 10.1016/S1360-1385(03)00011-6

Leyser, H. M., Pickett, F. B., Dharmasiri, S., and Estelle, M. (1996). Mutations in the AXR3 gene of Arabidopsis result in altered auxin response including ectopic expression from the SAUR-AC1 promoter. Plant J. 10, 403-413. doi: 10.1046/j.1365-313x.1996.10030403.x

Leyser, O. (2002). Molecular genetics of auxin signaling. Anu. Rev. Plant Biol. 53, 377-398. doi: 10.1146/annurev.arplant.53.100301.135227 
Li, G., and Xue, H.-W. (2007). Arabidopsis PLDzeta2 regulates vesicle trafficking and is required for auxin response. Plant Cell 19, 281-295. doi: 10.1105/tpc.106. 041426

Li, L., Xu, J., Xu, Z.-H., and Xue, H.-W. (2005). Brassinosteroids stimulate plant tropisms through modulation of polar auxin transport in Brassica and Arabidopsis. Plant Cell 17, 2738-2753. doi: 10.1105/tpc.105.034397

Li, P., Wind, J. J., Shi, X., Zhang, H., Hanson, J., Smeekens, S. C., et al. (2011). Fructose sensitivity is suppressed in Arabidopsis by the transcription factor ANAC089 lacking the membrane-bound domain. Proc. Natl. Acad. Sci. U.S.A. 108, 3436-3441. doi: 10.1073/pnas.1018665108

Li, Y., Lee, K. K., Walsh, S., Smith, C., Hadingham, S., Sorefan, K., et al. (2006). Establishing glucose- and ABA-regulated transcription networks in Arabidopsis by microarray analysis and promoter classification using a Relevance Vector Machine. Genome Res. 16, 414-427. doi: 10.1101/gr.4237406

Limbach, C., Hauslage, J., Schäfer, C., and Braun, M. (2005). How to activate a plant gravireceptor. Early mechanisms of gravity sensing studied in characean rhizoids during parabolic flights. Plant Physiol. 139, 1030-1040. doi: 10.1104/ pp.105.068106

Lin, D., Nagawa, S., Chen, J., Cao, L., Chen, X., Xu, T., et al. (2012). A ROP GTPasedependent auxin signaling pathway regulates the subcellular distribution of PIN2 in Arabidopsis roots. Curr. Biol. 22, 1319-1325. doi: 10.1016/j.cub.2012. 05.019

Löfke, C., Zwiewka, M., Heilmann, I., Van Montagu, M. C. E., Teichmann, T., and Friml, J. (2013). Asymmetric gibberellin signaling regulates vacuolar trafficking of PIN auxin transporters during root gravitropism. Proc. Natl. Acad. Sci. U.S.A. 110, 3627-3632. doi: 10.1073/pnas.1300107110

Lu, Y.-T., and Feldman, L. J. (1997). Light-regulated root gravitropism: a role for, and characterization of, a calcium/calmodulin-dependent protein kinase homolog. Planta 203, S91-S97. doi: 10.1007/PL00008121

Luschnig, C., Gaxiola, R. A., Grisafi, P., and Fink, G. R. (1998). EIR1, a root-specific protein involved in auxin transport, is required for gravitropism in Arabidopsis thaliana. Genes Dev. 12, 2175-2187. doi: 10.1101/gad.12.14.2175

Mancuso, S., Barlow, P. W., Volkmann, D., and Baluska, F. (2006). Actin turnovermediated gravity response in maize root apices: gravitropism of decapped roots implicates gravisensing outside of the root cap. Plant Signal. Behav. 1, 52-58. doi: $10.4161 /$ psb.1.2.2432

Masson, P. H., Tasaka, M., Morita, M. T., Guan, C., Chen, R., and Boonsirichai, K. (2002). Arabidopsis thaliana: a model for the study of root and shoot gravitropism. Arabidopsis Book 1, e0043. doi: 10.1199/tab.0043

Mei, Y., Jia, W.-J., Chu, Y.-J., and Xue, H.-W. (2012). Arabidopsis phosphatidylinositol monophosphate 5-kinase 2 is involved in root gravitropism through regulation of polar auxin transport by affecting the cycling of PIN proteins. Cell Res. 22, 581-597. doi: 10.1038/cr.2011.150

Mertens, R., and Weiler, E. (1983). Kinetic studies on the redistribution of endogenous growth regulators in gravireacting plant organs. Planta 158, 339-348. doi: 10.1007/BF00397336

Migliaccio, F., and Piconese, S. (2001). Spiralizations and tropisms in Arabidopsis roots. Trends Plant Sci. 6, 561-565. doi: 10.1016/S1360-1385(01)02152-5

Migliaccio, F., Tassone, P., and Fortunati, A. (2013). Circumnutation as an autonomous root movement in plants. Am. J. Bot. 100, 4-13. doi: 10.3732/ajb. 1200314

Miller, N. D., Parks, B. M., and Spalding, E. P. (2007). Computer-vision analysis of seedling responses to light and gravity. Plant J. 52, 374-381. doi: 10.1111/j. 1365-313X.2007.03237.x

Mirza, J. I. (1987). The effects of light and gravity on the horizontal curvature of roots of gravitropic and agravitropic Arabidopsis thaliana L. Plant Physiol. 83, 118-120. doi: 10.1104/pp.83.1.118

Mishra, B. S., Singh, M., Aggrawal, P., and Laxmi, A. (2009). Glucose and auxin signaling interaction in controlling Arabidopsis thaliana seedlings root growth and development. PLoS ONE 4:e4502. doi: 10.1371/journal.pone.0004502

Mo, M., Yokawa, K., Wan, Y., and Baluška, F. (2015). How and why do root apices sense light under the soil surface? Front. Plant Sci 6:775. doi: 10.3389/fpls.2015. 00775

Momonoki, Y. S. (1988). Asymmetric distribution of glucose and indole-3-acetylmyo-inositol in geostimulated Zea mays seedlings. Plant Physiol. 87, 751-756. doi: $10.1104 /$ pp.87.3.751

Monshausen, G. B., Miller, N. D., Murphy, A. S., and Gilroy, S. (2011). Dynamics of auxin-dependent $\mathrm{Ca} 2+$ and $\mathrm{pH}$ signaling in root growth revealed by integrating high-resolution imaging with automated computer vision-based analysis. Plant J. 65, 309-318. doi: 10.1111/j.1365-313X.2010.04423.x

Moore, B., Zhou, L., Rolland, F., Hall, Q., Cheng, W.-H., Liu, Y.-X., et al. (2003). Role of the Arabidopsis glucose sensor HXK1 in nutrient, light, and hormonal signaling. Science 300, 332-336. doi: 10.1126/science.1080585

Moore, R. (1990). Abscisic acid is not necessary for gravitropism in primary roots of Zea mays. Ann. Bot. 66, 281-283. doi: 10.1093/oxfordjournals.aob.a088026

Morita, M. T. (2010). Directional gravity sensing in gravitropism. Annu. Rev. Plant Biol. 61, 705-720. doi: 10.1146/annurev.arplant.043008.092042

Mullen, J. L., Wolverton, C., Ishikawa, H., and Evans, M. L. (2000). Kinetics of constant gravitropic stimulus responses in Arabidopsis roots using a feedback system. Plant Physiol. 123, 665-670. doi: 10.1104/pp.123.2.665

Okushima, Y., Overvoorde, P. J., Arima, K., Alonso, J. M., Chan, A., Chang, C., et al. (2005). Functional genomic analysis of the AUXIN RESPONSE FACTOR gene family members in Arabidopsis thaliana: unique and overlapping functions of ARF7 and ARF19. Plant Cell 17, 444-463. doi: 10.1105/tpc.104.028316

Oliva, M., and Dunand, C. (2007). Waving and skewing: how gravity and the surface of growth media affect root development in Arabidopsis. New Phytol. 176, 37-43. doi: 10.1111/j.1469-8137.2007.02184.x

Parry, G., Calderon-Villalobos, L., Prigge, M., Peret, B., Dharmasiri, S., Itoh, H., et al. (2009). Complex regulation of the TIR1/AFB family of auxin receptors. Proc. Natl. Acad. Sci. U.S.A. 106, 22540-22545. doi: 10.1073/pnas.0911967106

Perera, I. Y., Heilmann, I., Chang, S. C., Boss, W. F., and Kaufman, P. B. (2001). A role for inositol 1,4,5-trisphosphate in gravitropic signaling and the retention of cold-perceived gravistimulation of oat shoot pulvini. Plant Physiol. 125, 1499-1507. doi: 10.1104/pp.125.3.1499

Perera, I. Y., Hung, C.-Y., Brady, S., Muday, G. K., and Boss, W. F. (2006). A universal role for inositol 1,4,5-trisphosphate-mediated signaling in plant gravitropism. Plant Physiol. 140, 746-760. doi: 10.1104/pp.105.075119

Pernisová, M., Klíma, P., Horák, J., Válková, M., Malbeck, J., Souček, P., et al. (2009). Cytokinins modulate auxin-induced organogenesis in plants via regulation of the auxin efflux. Proc. Natl. Acad. Sci. U.S.A. 106, 3609-3614. doi: 10.1073/pnas.0811539106

Pernisova, M., Prat, T., Grones, P., Harustiakova, D., Matonohova, M., Spichal, L., et al. (2016). Cytokinins influence root gravitropism via differential regulation of auxin transporter expression and localization in Arabidopsis. New Phytol. 212, 497-509. doi: 10.1111/nph.14049

Perrineau, F., Wimalasekera, R., Effendi, Y., and Scherer, G. (2016). Inhibition of auxin transport and auxin signaling and treatment with far red light induces root coiling in the phospholipase-A mutant ppla-I-1. Significance for surface penetration? J. Plant Physiol. 196, 53-59. doi: 10.1016/j.jplph.2016. 03.010

Perry, S. E., and Keegstra, K. (1994). Envelope membrane proteins that interact with chloroplastic precursor proteins. Plant Cell 6, 93-105. doi: 10.1105/tpc. 6.1 .93

Philosoph-Hadas, S., Friedman, H., and Meir, S. (2005). Gravitropic bending and plant hormones. Vitam. Horm. 72, 31-78. doi: 10.1016/S0083-6729(05)72002-1

Pickett, F. B., Wilson, A. K., and Estelle, M. (1990). The aux1 mutation of Arabidopsis confers both auxin and ethylene resistance. Plant Physiol. 94, 1462-1466. doi: 10.1104/pp.94.3.1462

Price, J., Laxmi, A., St. Martin, S. K., and Jang, J.-C. (2004). Global transcription profiling reveals multiple sugar signal transduction mechanisms in Arabidopsis. Plant Cell 16, 2128-2150. doi: 10.1105/tpc.104.022616

Ramon, M., Rolland, F., and Sheen, J. (2008). Sugar sensing and signaling. Arabidopsis Book, 6:e0117. doi: 10.1199/tab.0117

Remington, D. L., Vision, T. J., Guilfoyle, T. J., and Reed, J. W. (2004). Contrasting modes of diversification in the Aux/IAA and ARF gene families. Plant Physiol. 135, 1738-1752. doi: 10.1104/pp.104.039669

Robert, H. S., and Offringa, R. (2008). Regulation of auxin transport polarity by AGC kinases. Curr. Opin. Plant Biol. 11, 495-502. doi: 10.1016/j.pbi.2008. 06.004

Rolland, F., Baena-Gonzalez, E., and Sheen, J. (2006). Sugar sensing and signaling in plants: conserved and novel mechanisms. Annu. Rev. Plant Biol. 57, 675-709. doi: 10.1146/annurev.arplant.57.032905.105441

Rolland, F., Moore, B., and Sheen, J. (2002). Sugar sensing and signaling in plants. Plant Cell 14, S185-S205.

Růzicka, K., Ljung, K., Vanneste, S., Podhorská, R., Beeckman, T., Friml, J., et al. (2007). Ethylene regulates root growth through effects on auxin biosynthesis 
and transport-dependent auxin distribution. Plant Cell 19, 21976-2212. doi: $10.1105 /$ tpc.107.052126

Salinas-Mondragon, R. E., Kajla, J. D., Perera, I. Y., Brown, C. S., and Sederoff, H. W. (2010). Role of inositol 1,4,5-triphosphate signalling in gravitropic and phototropic gene expression. Plant Cell Environ. 33, 2041-2055. doi: 10.1111/j. 1365-3040.2010.02204.x

Santisree, P., Nongmaithem, S., Vasuki, H., Sreelakshmi, Y., Ivanchenko, M. G., and Sharma, R. (2011). Tomato root penetration in soil requires a coaction between ethylene and auxin signaling. Plant Physiol. 156, 1424-1438. doi: 10.1104/pp. 111.177014

Sato, E. M., Hijazi, H., Bennett, M. J., Vissenberg, K., and Swarup, R. (2015). New insights into root gravitropic signalling. J. Exp. Bot. 66, 2155-2165. doi: $10.1093 / \mathrm{jxb} / \mathrm{eru} 515$

Schepetilnikov, M., Dimitrova, M., Mancera-Martínez, E., Geldreich, A., Keller, M., and Ryabova, L. A. (2013). TOR and S6K1 promote translation reinitiation of uORF-containing mRNAs via phosphorylation of eIF3h. EMBO J. 32, 1087-1102. doi: 10.1038/emboj.2013.61

Schwechheimer, C. (2008). Understanding gibberellic acid signaling-are we there yet? Curr. Opin. Plant Biol. 11, 9-15. doi: 10.1016/j.pbi.2007.10.011

Scott, A. C., and Allen, N. S. (1999). Changes in cytosolic pH within Arabidopsis root columella cells play a key role in the early signaling pathway for root gravitropism. Plant Physiol. 121, 1291-1298. doi: 10.1104/pp.121.4.1291

Sedbrook, J. C., Chen, R., and Masson, P. H. (1999). ARG1 (altered response to gravity) encodes a DnaJ-like protein that potentially interacts with the cytoskeleton. Proc. Natl. Acad. Sci. U.S.A. 96, 1140-1145. doi: 10.1073/pnas.96. 3.1140

Shih, H.-W., Depew, C. L., Miller, N. D., and Monshausen, G. B. (2015). The cyclic nucleotide-gated channel CNGC14 regulates root gravitropism in Arabidopsis thaliana. Curr. Biol. 25, 3119-3125. doi: 10.1016/j.cub.2015.10.025

Silva-Navas, J., Moreno-Risueno, M. A., Manzano, C., Téllez-Robledo, B., NavarroNeila, S., Carrasco, V., et al. (2016). Flavonols mediate root phototropism and growth through regulation of proliferation-to-differentiation transition. Plant Cell 28, 1372-1387. doi: 10.1105/tpc.15.00857

Simmons, C., Söll, D., and Migliaccio, F. (1995). Circumnutation and gravitropism cause root waving in Arabidopsis thaliana. J. Exp. Bot. 46, 143-150. doi: 10.1093/ jxb/46.1.143

Singh, M., Gupta, A., and Laxmi, A. (2014a). Glucose and phytohormone interplay in controlling root directional growth in Arabidopsis. Plant Signal. Behav. 9:e29219. doi: 10.4161/psb.29219

Singh, M., Gupta, A., and Laxmi, A. (2014b). Glucose control of root growth direction in Arabidopsis thaliana. J. Exp. Bot. 65, 2981-2993. doi: 10.1093/jxb/ eru146

Smeekens, S. (2000). Sugar-induced signal transduction in plants. Annu. Rev. Plant Physiol. Plant Mol. Biol. 51, 49-81. doi: 10.1146/annurev.arplant.51.1.49

Smeekens, S., Ma, J., Hanson, J., and Rolland, F. (2010). Sugar signals and molecular networks controlling plant growth. Curr. Opin. Plant Biol. 13, 274-279. doi: $10.1016 /$ j.pbi.2009.12.002

Smith, A. M., and Stitt, M. (2007). Coordination of carbon supply and plant growth. Plant Cell Environ. 30, 1126-1149. doi: 10.1111/j.1365-3040.2007.01708.x

Stanga, J. P., Boonsirichai, K., Sedbrook, J. C., Otegui, M. S., and Masson, P. H. (2009). A role for the TOC complex in Arabidopsis root gravitropism. Plant Physiol. 149, 1896-1905. doi: 10.1104/pp.109.135301

Staswick, P. E. (2009). The tryptophan conjugates of jasmonic and indole-3acetic acids are endogenous auxin inhibitors. Plant Physiol. 150, 1310-1321. doi: 10.1104/pp.109.138529

Staves, M. P. (1997). Cytoplasmic streaming and gravity sensing in Chara internodal cells. Planta 203, S79-S84. doi: 10.1007/PL00008119

Stinemetz, C. L., Kuzmanoff, K. M., Evans, M. L., and Jarrett, H. W. (1987). Correlation between calmodulin activity and gravitropic sensitivity in primary roots of maize. Plant Physiol. 84, 1337-1342. doi: 10.1104/pp.84.4.1337

Strohm, A. K., Baldwin, K. L., and Masson, P. H. (2012). Multiple roles for membrane-associated protein trafficking and signaling in gravitropism. Front. Plant Sci. 3:274. doi: 10.3389/fpls.2012.00274

Strohm, A. K., Barrett-Wilt, G. A., and Masson, P. H. (2014). A functional TOC complex contributes to gravity signal transduction in Arabidopsis. Front. Plant Sci. 5:148. doi: 10.3389/fpls.2014.00148

Sukumar, P., Edwards, K. S., Rahman, A., Delong, A., and Muday, G. K. (2009). PINOID kinase regulates root gravitropism through modulation of
PIN2-dependent basipetal auxin transport in Arabidopsis. Plant Physiol. 150, 722-735. doi: 10.1104/pp.108.131607

Swarup, R., and Péret, B. (2012). AUX/LAX family of auxin influx carriers-an overview. Front. Plant Sci. 3:225. doi: 10.3389/fpls.2012.00225

Szemenyei, H., Hannon, M., and Long, J. A. (2008). TOPLESS mediates auxin-dependent transcriptional repression during Arabidopsis embryogenesis. Science 319, 1384-1386. doi: 10.1126/science.1151461

Takahashi, N., Goto, N., Okada, K., and Takahashi, H. (2002). Hydrotropism in abscisic acid, wavy, and gravitropic mutants of Arabidopsis thaliana. Planta 216, 203-211. doi: 10.1007/s00425-002-0840-3

Taniguchi, Y. Y., Taniguchi, M., Tsuge, T., Oka, A., and Aoyama, T. (2010). Involvement of Arabidopsis thaliana phospholipase Dzeta2 in root hydrotropism through the suppression of root gravitropism. Planta 231, 491-497. doi: 10.1007/s00425-009-1052-x

Tasaka, M., Kato, T., and Fukaki, H. (1999). The endodermis and shoot gravitropism. Trends Plant Sci. 4, 103-107. doi: 10.1016/S1360-1385(99) 01376-X

Tepfer, D. A., and Bonnett, H. T. (1972). The role of phytochrome in the geotropic behavior of roots of Convolvulus arvensis. Planta 106, 311-324. doi: 10.1007/ BF00384768

Tian, Q., and Reed, J. W. (1999). Control of auxin-regulated root development by the Arabidopsis thaliana SHY2/IAA3 gene. Development 126, 711-721.

Toyota, M., and Gilroy, S. (2013). Gravitropism and mechanical signaling in plants. Am. J. Bot. 100, 111-125. doi: 10.3732/ajb.1200408

Tsugeki, R., and Fedoroff, N. V. (1999). Genetic ablation of root cap cells in Arabidopsis. Proc. Natl. Acad. Sci. U.S.A. 96, 12941-12946. doi: 10.1073/pnas. 96.22.12941

Vandenbrink, J. P., Herranz, R., Medina, F. J., Edelmann, R. E., and Kiss, J. Z. (2016). A novel blue-light phototropic response is revealed in roots of Arabidopsis thaliana in microgravity. Planta 244, 1201-1215. doi: 10.1007/ s00425-016-2581-8

Vandenbussche, F., Smalle, J., Le, J., Saibo, N. J. M., De Paepe, A., Chaerle, L., et al. (2003). The Arabidopsis mutant alh1 illustrates a cross talk between ethylene and auxin. Plant Physiol. 131, 1228-1238. doi: 10.1104/pp.010850

Vanneste, S., and Friml, J. (2009). Auxin: a trigger for change in plant development. Cell 136, 1005-1016. doi: 10.1016/j.cell.2009.03.001

Vanneste, S., and Friml, J. (2013). Calcium: the missing link in auxin action. Plants 2, 650-675. doi: 10.3390/plants2040650

Vitha, S., Yang, M., Sack, F. D., and Kiss, J. Z. (2007). Gravitropism in the starch excess mutant of Arabidopsis thaliana. Am. J. Bot. 94, 590-598. doi: 10.3732/ ajb. 94.4 .590

Volkmann, D., and Baluska, F. (1999). Actin cytoskeleton in plants: from transport networks to signaling networks. Microsc. Res. Tech. 47, 135-154. doi: 10.1002/ (SICI)1097-0029(19991015)47:2<135::AID-JEMT6>3.0.CO;2-1

von Wangenheim, D., Hauschild, R., Fendrych, M., Barone, V., and Friml, J. (2017). Live tracking of moving samples in confocal microscopy for vertically grown plant roots. bioRxiv, 6:e26792. doi: 10.7554/eLife.26792

Wan, Y., Jasik, J., Wang, L., Hao, H., Volkmann, D., Menzel, D., et al. (2012). The signal transducer NPH3 integrates the phototropin1 photosensor with PIN2based polar auxin transport in Arabidopsis root phototropism. Plant Cell 24, 551-565. doi: 10.1105/tpc.111.094284

Wang, H. Z., Yang, K. Z., Zou, J. J., Zhu, L. L., Xie, Z. D., Morita, M. T., et al. (2015). Transcriptional regulation of PIN genes by FOUR LIPS and MYB88 during Arabidopsis root gravitropism. Nat. Commun. 18:8822. doi: 10.1038/ ncomms 9822

Wang, Y., Lin, W.-H., Chen, X., and Xue, H.-W. (2009). The role of Arabidopsis 5PTase13 in root gravitropism through modulation of vesicle trafficking. Cell Res. 19:1191-1204. doi: 10.1038/cr.2009.105

Wayne, R., Staves, M. P., and Leopold, A. C. (1990). Gravity-dependent polarity of cytoplasmic streaming in Nitellopsis. Protoplasma 155, 43-57. doi: 10.1007/ BF01322614

Whitford, R., Fernandez, A., Tejos, R., Pérez, A. C., Kleine-Vehn, J., Vanneste, S., et al. (2012). GOLVEN secretory peptides regulate auxin carrier turnover during plant gravitropic responses. Dev. Cell 22, 678-685. doi: 10.1016/j.devcel.2012. 02.002

Wolverton, C., Mullen, J. L., Ishikawa, H., and Evans, M. L. (2002). Root gravitropism in response to a signal originating outside of the cap. Planta 215, 153-157. doi: 10.1007/s00425-001-0726-9 
Wolverton, C., Paya, A. M., and Toska, J. (2011). Root cap angle and gravitropic response rate are uncoupled in the Arabidopsis pgm-1 mutant. Physiol. Plant. 141, 373-382. doi: 10.1111/j.1399-3054.2010.01439.x

Xiong, Y., Mccormack, M., Li, L., Hall, Q., Xiang, C., and Sheen, J. (2013). Glucose-TOR signalling reprograms the transcriptome and activates meristems. Nature 496, 181-186. doi: 10.1038/nature12030

Yamada, M., Greenham, K., Prigge, M. J., Jensen, P. J., and Estelle, M. (2009). The TRANSPORT INHIBITOR RESPONSE2 gene is required for auxin synthesis and diverse aspects of plant development. Plant Physiol. 151, 168-179. doi: 10.1104/pp.109.138859

Yoder, T. L., Zheng, H. Q., Todd, P., and Staehelin, L. A. (2001). Amyloplast sedimentation dynamics in maize columella cells support a new model for the gravity-sensing apparatus of roots. Plant Physiol. 125 1045-1060. doi: 10.1104/ pp.125.2.1045

Yokawa, K., and Baluška, F. (2015). The TOR complex: an emergency switch for root behavior. Plant Cell Physiol. 57, 14-18. doi: 10.1093/pcp/pcv191

Yuen, C. Y. L., Sedbrook, J. C., Perrin, R. M., Carroll, K. L., and Masson, P. H. (2005). Loss-of-function mutations of ROOT HAIR DEFECTIVE3 suppress root waving, skewing, and epidermal cell file rotation in Arabidopsis. Plant Physiol. 138, 701-714. doi: 10.1104/pp.105.059774

Zhang, K. X., Xu, H. H., Yuan, T. T., Zhang, L., and Lu, Y. T. (2013). Blue-light-induced PIN3 polarization for root negative phototropic response in Arabidopsis. Plant J. 76, 308-321. doi: 10.1111/tpj.12298
Zheng, Z., Zou, J., Li, H., Xue, S., Wang, Y., and Le, J. (2015) Microrheological insights into the dynamics of amyloplasts in root gravity-sensing cells. Mol. Plant 8, 660-663. doi: 10.1016/j.molp.2014. 12.021

Zhou, Z.-Y., Zhang, C.-G., Wu, L., Zhang, C.-G., Chai, J., Wang, M., et al. (2011). Functional characterization of the CKRC1/TAA1 gene and dissection of hormonal actions in the Arabidopsis root. Plant J. 66, 516-527. doi: 10.1111/ j.1365-313X.2011.04509.x

Zou, J.-J., Zheng, Z.-Y., Xue, S., Li, H.-H., Wang, Y.-R., and Le, J. (2016). The role of Arabidopsis actin-related protein 3 in amyloplast sedimentation and polar auxin transport in root gravitropism. J. Exp. Bot. 67, 5325-5337. doi: $10.1093 /$ jxb/erw294

Conflict of Interest Statement: The authors declare that the research was conducted in the absence of any commercial or financial relationships that could be construed as a potential conflict of interest.

Copyright (c) 2017 Singh, Gupta and Laxmi. This is an open-access article distributed under the terms of the Creative Commons Attribution License (CC BY). The use, distribution or reproduction in other forums is permitted, provided the original author(s) or licensor are credited and that the original publication in this journal is cited, in accordance with accepted academic practice. No use, distribution or reproduction is permitted which does not comply with these terms. 